\title{
Geometric theory on the elasticity of bio-membranes
}

\author{
Z C Tu†‡* and Z C Ou-Yang†§ \\ $\dagger$ Institute of Theoretical Physics, Chinese Academy of Sciences, P. O. Box 2735 \\ Beijing 100080, China \\ $\ddagger$ Graduate School, Chinese Academy of Sciences, China \\ $\S$ Center for Advanced Study, Tsinghua University, Beijing 100084, China
}

\begin{abstract}
The purpose of this paper is to study the shapes and stabilities of biomembranes within the framework of exterior differential forms. After a brief review of the current status in theoretical and experimental studies on the shapes of biomembranes, a geometric scheme is proposed to discuss the shape equation of closed lipid bilayers, the shape equation and boundary conditions of open lipid bilayers and two-component membranes, the shape equation and in-plane strain equations of cell membranes with cross-linking structures, and the stabilities of closed lipid bilayers and cell membranes. The key point of this scheme is to deal with the variational problems on the surfaces embedded in three-dimensional Euclidean space by using exterior differential forms.
\end{abstract}

PACS numbers: 87.16.Dg, 02.30.Xx, 02.40.Hw

* Present address: Computational Material Science Center, National Institute for Materials Science, Tsukuba 305-0047, Japan. Email: tu.zhanchun@nims.go.jp 


\section{Introduction}

Cell membranes play crucial role in living movements. They consist of lipids, proteins and carbohydrates etc. There are many simplified models for cell membranes in history [1]. Among them, the widely accepted one is the fluid mosaic model proposed by Singer and Nicolson in 1972 [2]. In this model, a cell membrane is considered as a lipid bilayer where lipid molecules can move freely in the membrane surface like fluid, while proteins are embedded in the lipid bilayer. This model suggests that the shape of the cell membrane is determined by its lipid bilayer. Usually, the thickness of lipid bilayer is about 4 nanometers which is much less than the scale of the cell (about several micrometers). Therefore, we can use a geometrical surface to describe the lipid bilayer.

In 1973, Helfrich [3] proposed the curvature energy per unit area of the bilayer

$$
f_{c}=\left(k_{c} / 2\right)\left(2 H+c_{0}\right)^{2}+\bar{k} K,
$$

where $k_{c}$ and $\bar{k}$ are elastic constants; and $H, K, c_{0}$ are the mean, Gaussian, and spontaneous curvatures of the membrane surface, respectively. We can safely ignore the thermodynamic fluctuation of the curved bilayer at the room temperature because of $k_{c} \approx 10^{-19} \mathrm{~J} \gg k_{B} T[4,5]$, where $k_{B}$ is the Boltzmann factor and $T$ the room temperature. Based on Helfrich's curvature energy, the free energy of the closed bilayer under the osmotic pressure $p$ (the outer pressure minus the inner one) is written as

$$
\mathcal{F}_{H}=\int\left(f_{c}+\mu\right) d A+p \int d V
$$

where $d A$ is the area element, $\mu$ the surface tension of the bilayer, and $V$ the volume enclosed within the lipid bilayer. Starting with above free energy, many researchers studied the shapes of bilayers [6,7]. Especially, by taking the first order variation of the free energy, Ou-Yang et al. derived an equation to describe the equilibrium shape of the bilayer [8]:

$$
p-2 \mu H+k_{c}\left(2 H+c_{0}\right)\left(2 H^{2}-c_{0} H-2 K\right)+k_{c} \nabla^{2}(2 H)=0 .
$$

They also obtained that the threshold pressure for instability of spherical bilayer was $p_{c} \sim k_{c} / R^{3}$, where $R$ being the radius of spherical bilayer.

Recently, opening-up process of lipid bilayers by talin was observed by Saitoh et al. $[9,10]$, which arose the interest of studying the shape equation and boundary conditions of lipid bilayers with free exposed edges. Capovilla et al. first gave the shape equation and boundary conditions [11] of open lipid bilayers. They also discussed the mechanical meaning of these equations $[11,12]$. In recent paper, we also derived the shape equation and boundary conditions in different way-using exterior differential forms to deal with the variational problems on curved surfaces [13]. It is necessary to further develop this method because we have seen that it is much more concise than the tensor method in recent book [6] by one of the authors.

In fact, the structures of cell membranes are far more complex than the fluid mosaic model. The cross-linking structures exist in cell membranes where filaments of membrane skeleton link to proteins mosaicked in lipid bilayers [14]. It is worth 
discussing whether the cross-linking structures have effect on the shapes and stabilities of cell membranes.

In the following contents, both lipid bilayers and cell membranes are called biomembranes. We will fully develop our geometric method to study the shapes and stabilities of bio-membranes. Our method might not new for mathematicians who are familiar with the work by Griffiths and Bryant et al. $[15,16]$. Our method focuses on the application aspect, but the work by Griffiths and Bryant et al. emphasizes on the geometric meaning. Otherwise, we notice a nice review paper by Kamien [17], where he give an introduction to the classic differential geometry in soft materials. Here we will show that exterior differential forms not mentioned by Kamien might also be useful in the study of bio-membranes. This paper is organized as follows: In Sec 2, we briefly introduce the basic concepts in differential geometry and the variational theory of surfaces. In Sec 3, we deal with variational problems on a closed surface, and derive the shape equation of closed lipid bilayers, and then discuss the mechanical stabilities of spherical bilayers. In Sec4, we deal with variational problems on an open surface, and then derive the shape equation and boundary conditions of open lipid bilayers as well as two-component lipid bilayers. In Sec 5 , we derive the free energy of the cross-linking structure by analogy with the theory of rubber elasticity, and regard the free energy of the cell membrane as the sum of the free energy of the closed bilayer and that of cross-linking structure. The shape equation, in-plane stain equations, and mechanical stabilities of cell membranes are discussed by taking the first and second order variations of the total free energy. In Sec 6. we summarize the new results obtained in this paper.

\section{Mathematical preliminaries}

Here we assume that the readers are familiar with the basic concepts in differential geometry, such as manifold, differential form and Stokes theorem (see also Appendix A).

\subsection{Surfaces in three-dimensional Euclidean space, moving frame method}

At every point $P$ of a smooth and orientable surface $M$ in three-dimensional Euclidean space $\mathbb{E}^{3}$, as shown in Fig 1, we can construct an orthogonal system $\mathbf{e}_{1}, \mathbf{e}_{2}, \mathbf{e}_{3}$ such that $\mathbf{e}_{3}$ is the normal of the surface and $\mathbf{e}_{i} \cdot \mathbf{e}_{j}=\delta_{i j},(i, j=1,2,3)$. We call $\left\{P ; \mathbf{e}_{1}, \mathbf{e}_{2}, \mathbf{e}_{3}\right\}$ a moving frame. For the point in curve $C$, we let $\mathbf{e}_{1}$ be its tangent vector and $\mathbf{e}_{2}$ point to the inner point of $M$. The difference between two frames at point $P$ and $P^{\prime}$ (which is very close to $P$ ) is denoted by

$$
\begin{aligned}
& d \mathbf{r}=\lim _{P \rightarrow P^{\prime}} \overrightarrow{P P^{\prime}}=\omega_{1} \mathbf{e}_{1}+\omega_{2} \mathbf{e}_{2}, \\
& d \mathbf{e}_{i}=\omega_{i j} \mathbf{e}_{j} \quad(i=1,2,3),
\end{aligned}
$$

where $\omega_{1}, \omega_{2}$ and $\omega_{i j}(i, j=1,2,3)$ are 1 -forms.

It is easy to obtain $\omega_{i j}=-\omega_{j i}$ from $\mathbf{e}_{i} \cdot \mathbf{e}_{j}=\delta_{i j}$. Additionally, using $d d \mathbf{r}=0$ and $d d \mathbf{e}_{i}=0$, we obtain the structure equations of the surface:

$$
d \omega_{1}=\omega_{12} \wedge \omega_{2}
$$




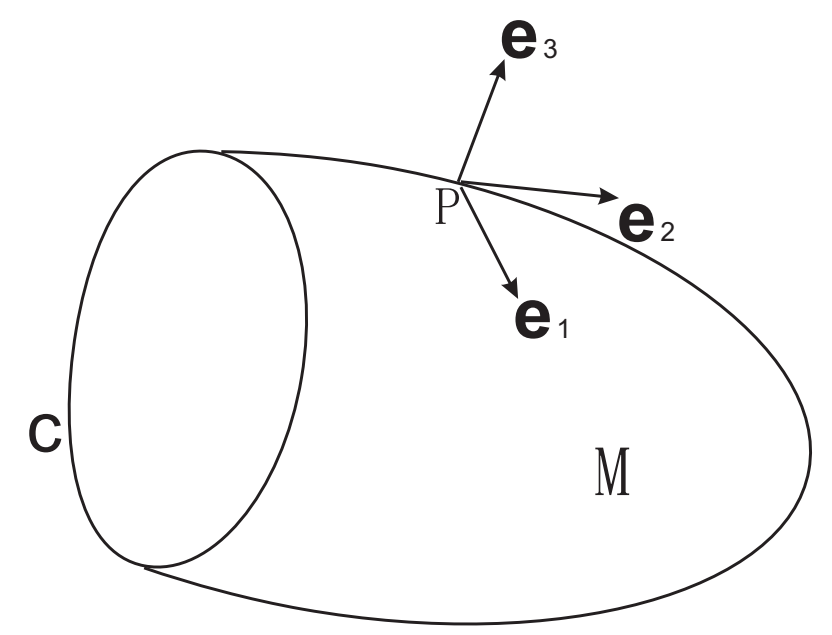

Figure 1. A smooth and orientable surface $M$ with an edge $C$.

$$
\begin{aligned}
& d \omega_{2}=\omega_{21} \wedge \omega_{1} ; \\
& \omega_{1} \wedge \omega_{13}+\omega_{2} \wedge \omega_{23}=0 ; \\
& d \omega_{i j}=\omega_{i k} \wedge \omega_{k j} \quad(i, j=1,2,3) .
\end{aligned}
$$

If we considering the Cartan Lemma, (8) suggests

$$
\omega_{13}=a \omega_{1}+b \omega_{2} \quad \text { and } \quad \omega_{23}=b \omega_{1}+c \omega_{2} .
$$

Thus we have [18]:

The area element: $d A=\omega_{1} \wedge \omega_{2}$,

The first fundamental form: $I=d \mathbf{r} \cdot d \mathbf{r}=\omega_{1}^{2}+\omega_{2}^{2}$,

The second fundamental form: $I I=-d \mathbf{r} \cdot d \mathbf{e}_{3}=a \omega_{1}^{2}+2 b \omega_{1} \omega_{2}+c \omega_{2}^{2}$,

The third fundamental form: $I I I=d \mathbf{e}_{3} \cdot d \mathbf{e}_{3}=\omega_{31}^{2}+\omega_{32}^{2}$,

Mean curvature: $H=(a+c) / 2$,

Gaussian curvature: $K=a c-b^{2}$.

\subsection{Hodge star * and Gauss mapping}

2.2.1. Hodge star $* \quad$ Here we just show the basic properties of Hodge star $*$ on surface $M$. So as to its general definition, please see Ref. [19].

If $g, h$ are functions defined on $2 \mathrm{D}$ smooth surface $M$, then the following formulas are valid:

$$
\begin{aligned}
& * f=f \omega_{1} \wedge \omega_{2} ; \\
& * \omega_{1}=\omega_{2}, * \omega_{2}=-\omega_{1} ; \\
& d * d f=\nabla^{2} f \omega_{1} \wedge \omega_{2},
\end{aligned}
$$

where $\nabla^{2}$ is Laplace-Beltrami operator. 
Geometric theory on the elasticity of bio-membranes

It is easy to obtain the second Green identity

$$
\int_{M}(f d * d h-h d * d f)=\int_{\partial M}(f * d h-h * d f)
$$

through Stokes theorem and the integration by parts. It follows that

$$
\int_{M} f d * d h=\int_{M} h d * d f
$$

if $M$ is a closed surface.

2.2.2. Gauss mapping The Gauss mapping $\mathcal{G}: M \rightarrow S^{2}$ is defined as $\mathcal{G}(\mathbf{r})=$ $\mathbf{e}_{3}(\mathbf{r})$, where $S^{2}$ is a unit sphere. It induces a linear mapping $\mathcal{G}^{\star}: \Lambda^{1} \rightarrow \Lambda^{1}$ such that:

(i) $\mathcal{G}^{\star} \omega_{1}=\omega_{13}, \mathcal{G}^{\star} \omega_{2}=\omega_{23}$;

(ii) if $d f=f_{1} \omega_{1}+f_{2} \omega_{2}$, then $\mathcal{G}^{\star} d f=f_{1} \mathcal{G}^{\star} \omega_{1}+f_{2} \mathcal{G}^{\star} \omega_{2}$.

Thus we can define a new differential operator $\tilde{d}=\mathcal{G}^{\star} d$. Obviously, if $d f=$ $f_{1} \omega_{1}+f_{2} \omega_{2}$, then $\tilde{d} f=f_{1} \omega_{13}+f_{2} \omega_{23}$. If define a new operator $\tilde{*}$ such that $\tilde{*} \omega_{13}=\omega_{23}$ and $\tilde{*} \omega_{23}=-\omega_{13}$, we have

Lemma 2.1 $\int_{M}(f d \tilde{*} \tilde{d} h-h d \tilde{*} \tilde{d} f)=\int_{\partial M}(f \tilde{*} \tilde{d} h-h \tilde{*} \tilde{d} f)$ for the smooth functions $f$ and $h$ on $M$.

Proof: Using the integration by parts and Stokes theorem, we obtain

$$
\begin{aligned}
\int_{M} f d \tilde{*} \tilde{d} h & =\int_{\partial M} f \tilde{*} \tilde{d} h-\int_{M} d f \wedge \tilde{*} \tilde{d} h \\
\int_{M} h d \tilde{*} \tilde{d} f & =\int_{\partial M} h \tilde{*} \tilde{d} f-\int_{M} d h \wedge \tilde{*} \tilde{d} f .
\end{aligned}
$$

Otherwise, if let $d f=f_{1} \omega_{1}+f_{2} \omega_{2}$ and $d h=h_{1} \omega_{1}+h_{2} \omega_{2}$, we can prove $d f \wedge \tilde{*} \tilde{d} h=d h \wedge \tilde{*} \tilde{d} f=\left[a f_{2} h_{2}+c f_{1} h_{1}-b\left(f_{1} h_{2}+f_{2} h_{1}\right)\right] \omega_{1} \wedge \omega_{2}$ through a few steps of calculations. Therefore, We can arrive at Lemma 2.1] by (19) minus (20) $\cdot$ -

It follows that

$$
\int_{M} f d \tilde{*} \tilde{d} h=\int_{M} h d \tilde{*} \tilde{d} f
$$

for a closed surface.

Because $d \tilde{*} \tilde{d} f$ is a 2 -form, we can define an operator $\nabla \cdot \tilde{\nabla}$ such that $d \tilde{*} \tilde{d} f=$ $\nabla \cdot \tilde{\nabla} f \omega_{1} \wedge \omega_{2}$.

\subsection{Variational theory of surface}

If let $M$ undergoes an infinitesimal deformation such that every point $\mathbf{r}$ of $M$ has a displacement $\delta \mathbf{r}$, we obtain a new surface $M^{\prime}=\left\{\mathbf{r}^{\prime} \mid \mathbf{r}^{\prime}=\mathbf{r}+\delta \mathbf{r}\right\}$. $\delta \mathbf{r}$ is called the variation of surface $M$ and expressed as

$$
\begin{aligned}
& \delta \mathbf{r}=\delta_{1} \mathbf{r}+\delta_{2} \mathbf{r}+\delta_{3} \mathbf{r}, \\
& \delta_{i} \mathbf{r}=\Omega_{i} \mathbf{e}_{i} \quad(i=1,2,3),
\end{aligned}
$$

where the repeated subindexes do not represent Einstein summation. 
Geometric theory on the elasticity of bio-membranes

Definition 2.1 If $f$ is a generalized function of $\mathbf{r}$ (including scalar function, vector function, and $r$-form dependent on point $\mathbf{r}$ ), define

$$
\delta_{i}^{(q)} f=(q !) \mathcal{L}^{(q)}\left[f\left(\mathbf{r}+\delta_{i} \mathbf{r}\right)-f(\mathbf{r})\right] \quad(i=1,2,3 ; q=1,2,3, \cdots),
$$

and the q-order variation of $f$

$$
\delta^{(q)} f=(q !) \mathcal{L}^{(q)}[f(\mathbf{r}+\delta \mathbf{r})-f(\mathbf{r})] \quad(q=1,2,3, \cdots),
$$

where $\mathcal{L}^{(q)}[\ldots]$ represents the terms of $\Omega_{1}^{q_{1}} \Omega_{2}^{q_{2}} \Omega_{3}^{q_{3}}$ in Taylor series of $[\cdots]$ with $q_{1}+q_{2}+$ $q_{3}=q$ and $q_{1}, q_{2}, q_{3}$ being non-negative integers.

It is easy to prove that:

(i) $\delta_{i}^{(q)}$ and $\delta^{(q)}(i=1,2,3 ; q=1,2, \cdots)$ are linear operators;

(ii) $\delta_{1}^{(1)}, \delta_{2}^{(1)}, \delta_{3}^{(1)}$ and $\delta^{(1)}$ are commutative with each other;

(iii) $\delta_{i}^{(q+1)}=\delta_{i}^{(1)} \delta_{i}^{(q)}$ and $\delta^{(q+1)}=\delta^{(1)} \delta^{(q)}$, thus we can safely replace $\delta_{i}^{(1)}, \delta_{i}^{(q)}, \delta^{(1)}$, and $\delta^{(q)}$ by $\delta_{i}, \delta_{i}^{q}, \delta$, and $\delta^{q}(q=2,3, \cdots)$, respectively;

(iv) For functions $f$ and $g, \delta_{i}[f(\mathbf{r}) \circ g(\mathbf{r})]=\delta_{i} f(\mathbf{r}) \circ g(\mathbf{r})+f(\mathbf{r}) \circ \delta_{i} g(\mathbf{r})$, where $\circ$ represents the ordinary production, vector production or exterior production;

(v) $\delta_{i} f[g(\mathbf{r})]=(\partial f / \partial g) \delta_{i} g$;

(vi) $\delta^{q}=\left(\delta_{1}+\delta_{2}+\delta_{3}\right)^{q}$, e.g. $\delta^{2}=\delta_{1}^{2}+\delta_{2}^{2}+\delta_{3}^{2}+2 \delta_{1} \delta_{2}+2 \delta_{2} \delta_{3}+2 \delta_{1} \delta_{3}$.

Due to the deformation of $M$, the vectors $\mathbf{e}_{1}, \mathbf{e}_{2}, \mathbf{e}_{3}$ are also changed. Denote their changes

$$
\delta_{l} \mathbf{e}_{i}=\Omega_{l i j} \mathbf{e}_{j},
$$

Obviously, $\mathbf{e}_{i} \cdot \mathbf{e}_{j}=\delta_{i j}$ implies $\Omega_{l i j}=-\Omega_{l j i}$. Because $\delta_{1}, \delta_{2}$ and $\delta_{3}$ are linear mappings from $M$ to $M^{\prime}$, they are commutative with exterior differential operator $d$ [18]. Therefore, using $d \delta_{l} \mathbf{r}=\delta_{l} d \mathbf{r}$ and $d \delta_{l} \mathbf{e}_{j}=\delta_{l} d \mathbf{e}_{j}$, we arrive at

$$
\begin{aligned}
& \delta_{1} \omega_{1}=d \Omega_{1}-\omega_{2} \Omega_{121}, \\
& \delta_{1} \omega_{2}=\Omega_{1} \omega_{12}-\omega_{1} \Omega_{112}, \\
& \Omega_{113}=a \Omega_{1}, \quad \Omega_{123}=b \Omega_{1} ; \\
& \delta_{2} \omega_{1}=\Omega_{2} \omega_{21}-\omega_{2} \Omega_{221}, \\
& \delta_{2} \omega_{2}=d \Omega_{2}-\omega_{1} \Omega_{212}, \\
& \Omega_{213}=b \Omega_{2}, \quad \Omega_{223}=c \Omega_{2} ; \\
& \delta_{3} \omega_{1}=\Omega_{3} \omega_{31}-\omega_{2} \Omega_{321}, \\
& \delta_{3} \omega_{2}=\Omega_{3} \omega_{32}-\omega_{1} \Omega_{312}, \\
& d \Omega_{3}=\Omega_{313} \omega_{1}+\Omega_{323} \omega_{2} ; \\
& \delta_{l} \omega_{i j}=d \Omega_{l i j}+\Omega_{l i k} \omega_{k j}-\omega_{i k} \Omega_{l k j} .
\end{aligned}
$$

Above equations (27) $\sim(36)$ are the fundamental equations in our paper and have not existed in previous mathematical literature [15] and [16]. Otherwise, it is easy to deduce that $\delta_{i} \tilde{d} f=\tilde{d} \delta_{i} f(i=1,2,3)$ for function $f$. 
Geometric theory on the elasticity of bio-membranes

\section{Closed lipid bilayers}

In this section, we will discuss the equilibrium shapes and mechanical stabilities of closed lipid bilayers. We just consider the closed surface in this section.

\subsection{First order variational problems on a closed surface}

In this subsection, we will discuss the first order variation of the functional

$$
\mathcal{F}=\int_{M} \mathcal{E}(2 H[\mathbf{r}], K[\mathbf{r}]) d A+p \int_{V} d V
$$

where $H$ and $K$ are mean and gaussian curvatures at point $\mathbf{r}$ in surface $M . p$ is a constant and $V$ be the volume enclosed within the surface.

According to the variational theory of surface in Sec 2, we have $\delta \mathcal{F}=\delta_{1} \mathcal{F}+\delta_{2} \mathcal{F}+$ $\delta_{3} \mathcal{F}$. Therefore, the next tasks are to calculate $\delta_{1} \mathcal{F}, \delta_{2} \mathcal{F}$ and $\delta_{3} \mathcal{F}$, respectively.

3.1.1. Calculation of $\delta_{3} \mathcal{F} \quad$ Here, we will briefly prove 4 Lemmas and a theorem. Above all, denote

$$
\mathcal{F}_{e}=\int_{M} \mathcal{E}(2 H[\mathbf{r}], K[\mathbf{r}]) d A
$$

Lemma $3.1 \delta_{3} d A=-(2 H) \Omega_{3} d A$.

Proof: $\delta_{3} d A=\delta_{3}\left(\omega_{1} \wedge \omega_{2}\right)=\delta_{3} \omega_{1} \wedge \omega_{2}+\omega_{1} \wedge \delta_{3} \omega_{2}$. Considering (10), (12), (33) and (34), we arrive at this Lemma.

Lemma $3.2 \delta_{3}(2 H) d A=2\left(2 H^{2}-K\right) \Omega_{3} d A+d * d \Omega_{3}$.

Proof: $\delta_{3}(2 H) d A=\delta_{3} a \omega_{1} \wedge \omega_{2}+\delta_{3} c \omega_{1} \wedge \omega_{2}$. Let $\delta_{3}$ acts on (10), we have

$$
\begin{aligned}
& \delta_{3} \omega_{13}=\delta_{3} a \omega_{1}+a \delta_{3} \omega_{1}+\delta_{3} b \omega_{2}+b \delta_{3} \omega_{2}, \\
& \delta_{3} \omega_{23}=\delta_{3} b \omega_{1}+b \delta_{3} \omega_{1}+\delta_{3} c \omega_{2}+c \delta_{3} \omega_{2} .
\end{aligned}
$$

If considering (12), (13), (15), (33) (36), we arrive at this Lemma. I

Lemma $3.3 \delta_{3} K d A=2 K H \Omega_{3} d A+d \tilde{*} \tilde{d} \Omega_{3}$.

Proof: Theorem Egregium (see Appendix C) implies that $\delta_{3} K d A=-\delta_{3} d \omega_{12}-K \delta_{3} d A=$ $-d \delta_{3} \omega_{12}-K \delta_{3} d A$. We will arrive at this Lemma from (36) and Lemma 3.1 as well as the discussions in Sec 2.2.2

Theorem $3.1 \delta_{3} \mathcal{F}_{e}=\int_{M}\left[\left(\nabla^{2}+4 H^{2}-2 K\right) \frac{\partial \mathcal{E}}{\partial(2 H)}+(\nabla \cdot \tilde{\nabla}+2 K H) \frac{\partial \mathcal{E}}{\partial K}-2 H \mathcal{E}\right] \Omega_{3} d A$.

Proof: Above all, we have

$$
\begin{aligned}
\delta_{3} \mathcal{F}_{e} & =\int_{M} \delta_{3} \mathcal{E} d A+\int_{M} \mathcal{E} \delta_{3} A \\
& =\int_{M} \frac{\partial \mathcal{E}}{\partial(2 H)} \delta_{3}(2 H) d A+\int_{M} \frac{\partial \mathcal{E}}{\partial K} \delta_{3} K d A+\int_{M} \mathcal{E} \delta_{3} d A .
\end{aligned}
$$


Geometric theory on the elasticity of bio-membranes

By using Lemmas 3.1, 3.2, 3.3, we obtain

$$
\begin{aligned}
\delta_{3} \mathcal{F}_{e} & =\int_{M}\left[\left(4 H^{2}-2 K\right) \frac{\partial \mathcal{E}}{\partial(2 H)}+2 K H \frac{\partial \mathcal{E}}{\partial K}-2 H \mathcal{E}\right] \Omega_{3} d A \\
& +\int_{M}\left[\frac{\partial \mathcal{E}}{\partial(2 H)} d * d \Omega_{3}+\frac{\partial \mathcal{E}}{\partial K} d \tilde{*} \tilde{d} \Omega_{3}\right] .
\end{aligned}
$$

For the closed surface $M$, we arrive at this theorem by considering (18) and (21).

Lemma $3.4 \delta_{3} \int_{V} d V=\int_{M} \Omega_{3} d A$.

Proof: Because $M$ is a closed surface in $\mathbb{E}^{3}$, Stokes theorem (see Appendix A) implies $\int_{V} 3 d V=\int_{V} \nabla \cdot \mathbf{r} d V=\int_{\partial V} \mathbf{r} \cdot \mathbf{n} d A$, thus

$$
\begin{aligned}
\delta_{3} \int_{V} d V & =\frac{1}{3} \int_{M} \delta_{3}\left[\mathbf{r} \cdot \mathbf{e}_{3}\left(\omega_{1} \wedge \omega_{2}\right)\right] \\
& =\frac{1}{3} \int_{M}\left[\delta_{3} \mathbf{r} \cdot \mathbf{e}_{3}\left(\omega_{1} \wedge \omega_{2}\right)+\mathbf{r} \cdot \delta_{3} \mathbf{e}_{3}\left(\omega_{1} \wedge \omega_{2}\right)+\mathbf{r} \cdot \mathbf{e}_{3} \delta_{3}\left(\omega_{1} \wedge \omega_{2}\right)\right] .
\end{aligned}
$$

From (33) (35), we obtain

$$
\begin{aligned}
& \delta_{3} \mathbf{r} \cdot \mathbf{e}_{3}\left(\omega_{1} \wedge \omega_{2}\right)=\Omega_{3} \omega_{1} \wedge \omega_{2}, \\
& \mathbf{r} \cdot \mathbf{e}_{3} \delta_{3}\left(\omega_{1} \wedge \omega_{2}\right)=\mathbf{r} \cdot \mathbf{e}_{3}(-2 H) \Omega_{3} \omega_{1} \wedge \omega_{2}, \\
& \mathbf{r} \cdot \delta_{3} \mathbf{e}_{3}\left(\omega_{1} \wedge \omega_{2}\right)=-d \Omega_{3} \wedge\left(-\mathbf{r} \cdot \mathbf{e}_{2} \omega_{1}+\mathbf{r} \cdot \mathbf{e}_{1} \omega_{2}\right) .
\end{aligned}
$$

By using the integration by parts and Stokes theorem, we have

$$
\begin{aligned}
-\int_{M} d \Omega_{3} \wedge\left(-\mathbf{r} \cdot \mathbf{e}_{2} \omega_{1}+\mathbf{r} \cdot \mathbf{e}_{1} \omega_{2}\right) & =\int_{M} \Omega_{3} d\left(-\mathbf{r} \cdot \mathbf{e}_{2} \omega_{1}+\mathbf{r} \cdot \mathbf{e}_{1} \omega_{2}\right) \\
& =\int_{M} \Omega_{3}\left[2+\mathbf{r} \cdot \mathbf{e}_{3}(2 H)\right] \omega_{1} \wedge \omega_{2} .
\end{aligned}
$$

Therefore, we will arrive at $\delta_{3} \int_{V} d V=\int_{M} \Omega_{3} d A$ by using (40) (44). व

\subsubsection{Calculation of $\delta_{1} \mathcal{F}$ and $\delta_{2} \mathcal{F}$}

Theorem $3.2 \delta_{1} \mathcal{F} \equiv 0$ and $\delta_{2} \mathcal{F} \equiv 0$.

Proof: We obtain

$$
d b \wedge \omega_{1}+2 b d \omega_{1}=(a-c) d \omega_{2}-d c \wedge \omega_{2} .
$$

from (9) and (10).

Using (27) (29), (36), and (45), we arrive at

$$
\begin{aligned}
& \delta_{1}\left(\omega_{1} \wedge \omega_{2}\right)=d\left(\Omega_{1} \omega_{2}\right), \\
& \delta_{1}(2 H) \omega_{1} \wedge \omega_{2}=d(2 H) \wedge \omega_{2} \Omega_{1}
\end{aligned}
$$

through a few calculations.

By analogy with the proof of Lemma 3.3, we can prove that

$$
\delta_{1} K \omega_{1} \wedge \omega_{2}=d K \wedge \Omega_{1} \omega_{2} .
$$


Geometric theory on the elasticity of bio-membranes

Therefore, we have

$$
\begin{aligned}
\delta_{1} \mathcal{F}_{e} & =\int_{M}\left[\frac{\partial \mathcal{E}}{\partial(2 H)} \delta_{1}(2 H) \omega_{1} \wedge \omega_{2}+\frac{\partial \mathcal{E}}{\partial K} \delta_{1} K \omega_{1} \wedge \omega_{2}+\mathcal{E} \delta_{1}\left(\omega_{1} \wedge \omega_{2}\right)\right] \\
& =\int_{M} d\left(\mathcal{E} \omega_{2} \Omega_{1}\right) .
\end{aligned}
$$

Similarly, we can obtain

$$
\delta_{2} \mathcal{F}_{e}=-\int_{M} d\left(\mathcal{E} \omega_{1} \Omega_{2}\right) .
$$

Otherwise, it is not hard to obtain $\delta_{1} \int_{V} d V=\int_{M} d\left(\mathbf{r} \cdot \mathbf{e}_{3} \omega_{2} \Omega_{1}\right)$ and $\delta_{2} \int_{V} d V=$ $-\int_{M} d\left(\mathbf{r} \cdot \mathbf{e}_{3} \omega_{1} \Omega_{2}\right)$.

Therefore, $\delta_{1} \mathcal{F}=\delta_{2} \mathcal{F} \equiv 0$ because $M$ is a closed surface.

\subsubsection{Euler-Lagrange equation}

Till now, we can obtain

$$
\delta \mathcal{F}=\int_{M}\left[\left(\nabla^{2}+4 H^{2}-2 K\right) \frac{\partial \mathcal{E}}{\partial(2 H)}+(\nabla \cdot \tilde{\nabla}+2 K H) \frac{\partial \mathcal{E}}{\partial K}-2 H \mathcal{E}+p\right] \Omega_{3} d A .
$$

Thus the Euler-Lagrange equation corresponding to the functional $\mathcal{F}$ is:

$$
\left[\left(\nabla^{2}+4 H^{2}-2 K\right) \frac{\partial}{\partial(2 H)}+(\nabla \cdot \tilde{\nabla}+2 K H) \frac{\partial}{\partial K}-2 H\right] \mathcal{E}(2 H, K)+p=0 .
$$

The similar equation is first found in Ref. [20].

\subsection{Second order variation}

In this subsection, we discuss the second order variation of functional (37) . This problem was also studied by Capovilla and Guven in recent paper [21]. Because $\delta_{1} \mathcal{F}=\delta_{2} \mathcal{F} \equiv 0$ for closed surface $M$, we have $\delta \delta_{1} \mathcal{F}=\delta \delta_{2} \mathcal{F}=0$, and $\delta^{2} \mathcal{F}=\delta \delta_{3} \mathcal{F}=\delta_{3}^{2} \mathcal{F}$.

Form (39) and Lemma 3.4, we obtain

$$
\begin{aligned}
\delta^{2} \mathcal{F}= & \delta_{3} \int_{M}\left[\left(4 H^{2}-2 K\right) \frac{\partial \mathcal{E}}{\partial(2 H)}+(2 K H) \frac{\partial \mathcal{E}}{\partial K}-2 H \mathcal{E}+p\right] \Omega_{3} d A \\
& +\delta_{3} \int_{M} \frac{\partial \mathcal{E}}{\partial(2 H)} d * d \Omega_{3}+\delta_{3} \int_{M} \frac{\partial \mathcal{E}}{\partial K} d \tilde{*} \tilde{d} \Omega_{3} \\
= & \int_{M} \delta_{3}\left[\left(4 H^{2}-2 K\right) \frac{\partial \mathcal{E}}{\partial(2 H)}+(2 K H) \frac{\partial \mathcal{E}}{\partial K}-2 H \mathcal{E}+p\right] \Omega_{3} d A \\
& +\int_{M}\left[\left(4 H^{2}-2 K\right) \frac{\partial \mathcal{E}}{\partial(2 H)}+(2 K H) \frac{\partial \mathcal{E}}{\partial K}-2 H \mathcal{E}+p\right] \Omega_{3} \delta_{3} d A \\
& +\int_{M} \delta_{3}\left[\frac{\partial \mathcal{E}}{\partial(2 H)}\right] d * d \Omega_{3}+\frac{\partial \mathcal{E}}{\partial(2 H)} \delta_{3}\left(d * d \Omega_{3}\right) \\
& +\int_{M} \delta_{3}\left(\frac{\partial \mathcal{E}}{\partial K}\right) d \tilde{*} \tilde{d} \Omega_{3}+\frac{\partial \mathcal{E}}{\partial K} \delta_{3}\left(d \tilde{*} \tilde{d} \Omega_{3}\right) .
\end{aligned}
$$

Please notice that $\Omega_{3}$ can freely come into and out of the expressions acted by the operator $\delta_{3}$. 
Geometric theory on the elasticity of bio-membranes

Lemma 3.5 For every function $f, \delta_{3} d * d f=d * d \delta_{3} f+d\left(2 H \Omega_{3} * d f\right)-2 d\left(\Omega_{3} \tilde{*} \tilde{d} f\right)$.

Proof: Let $d f=f_{1} \omega_{1}+f_{2} \omega_{2}$, we have $* d f=f_{1} \omega_{2}-f_{2} \omega_{1}, \tilde{d} f=f_{1} \omega_{13}+f_{2} \omega_{23}$ and $\tilde{*} \tilde{d} f=f_{1} \omega_{23}-f_{2} \omega_{13}$. By using (33) and (34), we have

$$
\begin{aligned}
& \delta_{3} * d f=\left(\delta_{3} f_{1} \omega_{2}-\delta_{3} f_{2} \omega_{1}\right)-\Omega_{312} d f+\Omega_{3}\left[f_{2}\left(a \omega_{1}+b \omega_{2}\right)-f_{1}\left(b \omega_{1}+c \omega_{2}\right)\right], \\
& * \delta_{3} d f=\left(\delta_{3} f_{1} \omega_{2}-\delta_{3} f_{2} \omega_{1}\right)-\Omega_{312} d f+\Omega_{3}\left[f_{1}\left(b \omega_{1}-a \omega_{2}\right)+f_{2}\left(c \omega_{1}-b \omega_{2}\right)\right], \\
& \delta_{3} * d f-* \delta_{3} d f=2 H \Omega_{3} * d f-2 \Omega_{3} \tilde{d} f .
\end{aligned}
$$

Using the operator $d$ to act on both sides of (154) and noticing the commutativity of $d$ and $\delta_{3}$, we arrive at this Lemma.

Lemma 3.6 For every function $f, \delta_{3} d \tilde{*} \tilde{d} f=d\left[\delta_{3}(2 H) * d f+2 H \delta_{3} * d f+2 K \Omega_{3} * d f-\right.$ $\left.2 H \Omega_{3} * \tilde{d} f-* \tilde{d} \delta_{3} f\right]$.

Proof: Similar to the proof of Lemma 3.5, we have

$$
\begin{aligned}
& \delta_{3} * \tilde{d} f=\delta_{3}\left(a f_{1}+b f_{2}\right) \omega_{2}-\delta_{3}\left(b f_{1}+c f_{2}\right) \omega_{1}-K \Omega_{3} * d f-\Omega_{312} \tilde{d} f, \\
& * \delta_{3} \tilde{d} f=\delta_{3}\left(a f_{1}+b f_{2}\right) \omega_{2}-\delta_{3}\left(b f_{1}+c f_{2}\right) \omega_{1}-2 H \Omega_{3} * \tilde{d} f+K \Omega_{3} * d f-\Omega_{312} \tilde{d} f .
\end{aligned}
$$

The difference of above two equations gives

$$
* \delta_{3} \tilde{d} f-\delta_{3} * \tilde{d} f=2 K \Omega_{3} * d f-2 H \Omega_{3} * \tilde{d} f .
$$

Otherwise, It is easy to see

$$
* \tilde{d} f+\tilde{*} \tilde{d} f=2 H * d f .
$$

Using $d \delta_{3}$ to act on both sides of (156) and $d$ to act on both sides of (155), considering the commutative relations $\tilde{d} \delta_{3}=\delta_{3} \tilde{d}$ and $d \delta_{3}=\delta_{3} d$, we arrive at this Lemma.

If $d f=f_{1} \omega_{1}+f_{2} \omega_{2}$, we define $\nabla f=f_{1} \mathbf{e}_{1}+f_{2} \mathbf{e}_{2}, \bar{\nabla} f=\left(a f_{1}+b f_{2}\right) \mathbf{e}_{1}+\left(b f_{1}+c f_{2}\right) \mathbf{e}_{2}$, $\tilde{\nabla} f=\left(c f_{1}-b f_{2}\right) \mathbf{e}_{1}+\left(a f_{2}-b f_{1}\right) \mathbf{e}_{2}$ and $d * \tilde{d} f=(\nabla \cdot \bar{\nabla}) f \omega_{1} \wedge \omega_{2}$. It follows that, for function $f$ and $g$,

$$
\begin{aligned}
& \tilde{\nabla} f+\bar{\nabla} f=2 H \nabla f \\
& d f \wedge * d g=(\nabla f \cdot \nabla g) \omega_{1} \wedge \omega_{2}, \\
& d f \wedge * \tilde{d} g=(\nabla f \cdot \bar{\nabla} g) \omega_{1} \wedge \omega_{2}, \\
& d f \wedge \tilde{*} \tilde{d} g=(\nabla f \cdot \tilde{\nabla} g) \omega_{1} \wedge \omega_{2} .
\end{aligned}
$$

Remark 3.1 The tensor expressions of $\nabla, \bar{\nabla}, \tilde{\nabla}, \nabla^{2}, \nabla \cdot \bar{\nabla}, \nabla \cdot \tilde{\nabla}$ are developed in Appendix D.

Theorem 3.3 The second order variation of functional (37) is

$$
\begin{aligned}
\delta^{2} \mathcal{F}= & \int_{M} \Omega_{3}^{2}\left[\left(4 H^{2}-2 K\right)^{2} \frac{\partial^{2} \mathcal{E}}{\partial(2 H)^{2}}-4 K H \frac{\partial \mathcal{E}}{\partial(2 H)}-2 K^{2} \frac{\partial \mathcal{E}}{\partial K}\right. \\
& \left.+4 K H\left(4 H^{2}-2 K\right) \frac{\partial^{2} \mathcal{E}}{\partial(2 H) \partial K}+4 K^{2} H^{2} \frac{\partial^{2} \mathcal{E}}{\partial K^{2}}+2 K \mathcal{E}-2 H p\right] d A \\
+ & \int_{M} \Omega_{3} \nabla^{2} \Omega_{3}\left[4 H \frac{\partial \mathcal{E}}{\partial(2 H)}+4\left(2 H^{2}-K\right) \frac{\partial^{2} \mathcal{E}}{\partial(2 H)^{2}}+K \frac{\partial \mathcal{E}}{\partial K}\right.
\end{aligned}
$$


Geometric theory on the elasticity of bio-membranes

$$
\begin{aligned}
& \left.\quad+4 H K \frac{\partial^{2} \mathcal{E}}{\partial K \partial(2 H)}-\mathcal{E}+8 H^{2} \frac{\partial \mathcal{E}}{\partial K}\right] d A \\
& +\int_{M} \Omega_{3} \nabla \cdot \tilde{\nabla} \Omega_{3}\left[4\left(2 H^{2}-K\right) \frac{\partial^{2} \mathcal{E}}{\partial(2 H) \partial K}-4 \frac{\partial \mathcal{E}}{\partial(2 H)}\right. \\
& \left.\quad+4 H K \frac{\partial^{2} \mathcal{E}}{\partial K^{2}}-4 H \frac{\partial \mathcal{E}}{\partial K}\right] d A \\
& +\int_{M}\left(\nabla^{2} \Omega_{3}\right)^{2}\left[\frac{\partial^{2} \mathcal{E}}{\partial(2 H)^{2}}+\frac{\partial \mathcal{E}}{\partial K}\right] d A \\
& +\int_{M}\left[\frac{2 \partial^{2} \mathcal{E}}{\partial(2 H) \partial K} \nabla^{2} \Omega_{3} \nabla \cdot \tilde{\nabla} \Omega_{3}+\frac{\partial \mathcal{E}}{\partial(2 H)} \nabla\left(2 H \Omega_{3}\right) \cdot \nabla \Omega_{3}\right] d A \\
& +\int_{M}\left[\frac{\partial^{2} \mathcal{E}}{\partial K^{2}}\left(\nabla \cdot \tilde{\nabla} \Omega_{3}\right)^{2}-\frac{2 \partial \mathcal{E}}{\partial(2 H)} \nabla \Omega_{3} \cdot \tilde{\nabla} \Omega_{3} d A\right] \\
& +\int_{M} \frac{\partial \mathcal{E}}{\partial K}\left[\nabla\left(8 H^{2} \Omega_{3}+\nabla^{2} \Omega_{3}\right) \cdot \nabla \Omega_{3}-\nabla\left(4 H \Omega_{3}\right) \cdot \tilde{\nabla} \Omega_{3}-4 H \Omega_{3} \nabla \cdot \tilde{\nabla} \Omega_{3}\right. \\
& \left.\quad-\nabla\left(2 H \Omega_{3}\right) \cdot \bar{\nabla} \Omega_{3}-2 H \Omega_{3} \nabla \cdot \bar{\nabla} \Omega_{3}\right] d A .
\end{aligned}
$$

Proof: Replacing $f$ by $\Omega_{3}$ in Lemma 3.5 and 3.6. and noticing that $\Omega_{3}$ is similar to a constant relative to $\delta_{3}$, we have

$$
\begin{aligned}
& \delta_{3} d * d \Omega_{3}=\left[\nabla\left(2 H \Omega_{3}\right) \cdot \nabla \Omega_{3}+2 H \Omega_{3} \nabla^{2} \Omega_{3}-2 \nabla \Omega_{3} \cdot \tilde{\nabla} \Omega_{3}-2 \Omega_{3} \nabla \cdot \tilde{\nabla} \Omega_{3}\right] d A, \\
& \delta_{3} d \tilde{*} \tilde{d} \Omega_{3}=\left[\nabla\left(8 H^{2} \Omega_{3}+\nabla^{2} \Omega_{3}\right) \cdot \nabla \Omega_{3}+\left(8 H^{2} \Omega_{3}+\nabla^{2} \Omega_{3}\right) \nabla^{2} \Omega_{3}\right. \\
& \left.\quad-\nabla\left(4 H \Omega_{3}\right) \cdot \tilde{\nabla} \Omega_{3}-4 H \Omega_{3} \nabla \cdot \tilde{\nabla} \Omega_{3}-\nabla\left(2 H \Omega_{3}\right) \cdot \bar{\nabla} \Omega_{3}-2 H \Omega_{3} \nabla \cdot \bar{\nabla} \Omega_{3}\right] d A .
\end{aligned}
$$

Substituting them into (53) and using Lemmas 3.2 and 3.3, we can arrive at this theorem through expatiatory calculations. ब

In particular, if $\partial \mathcal{E} / \partial K=\bar{k}$ being a constant, (153) is simplified to

$$
\begin{aligned}
\delta^{2} \mathcal{F}= & \int_{M} \Omega_{3}^{2}\left[\left(4 H^{2}-2 K\right)^{2} \frac{\partial^{2} \mathcal{E}_{H}}{\partial(2 H)^{2}}-4 H K \frac{\partial \mathcal{E}_{H}}{\partial(2 H)}+2 K \mathcal{E}_{H}-2 H p\right] d A \\
& +\int_{M} \Omega_{3} \nabla^{2} \Omega_{3}\left[4 H \frac{\partial \mathcal{E}_{H}}{\partial(2 H)}+4\left(2 H^{2}-K\right) \frac{\partial^{2} \mathcal{E}_{H}}{\partial(2 H)^{2}}-\mathcal{E}_{H}\right] d A \\
& -\int_{M} \frac{4 \partial \mathcal{E}_{H}}{\partial(2 H)} \Omega_{3} \nabla \cdot \tilde{\nabla} \Omega_{3} d A+\int_{M} \frac{\partial^{2} \mathcal{E}_{H}}{\partial(2 H)^{2}}\left(\nabla^{2} \Omega_{3}\right)^{2} d A \\
& +\int_{M} \frac{\partial \mathcal{E}_{H}}{\partial(2 H)}\left[\nabla\left(2 H \Omega_{3}\right) \cdot \nabla \Omega_{3}-2 \nabla \Omega_{3} \cdot \tilde{\nabla} \Omega_{3}\right] d A
\end{aligned}
$$

where $\mathcal{E}_{H}=\mathcal{E}-\bar{k} K$.

\subsection{Shape equation of closed lipid bilayers}

Now, Let us turn to the shape equation of closed lipid bilayers. We take the free energy of closed lipid bilayer under the osmotic pressure as (2). Substituting $\mathcal{E}=$ $\left(k_{c} / 2\right)\left(2 H+c_{0}\right)^{2}+\bar{k} K+\mu$ into (52), we obtain the shape equation (33). This equation is the fourth order nonlinear equation. It is not easy to obtain its special solutions. We 
will give three typical analytical solutions as follows. Some new important results on it can be found in recent paper by Landolfi [22].

\subsubsection{Constant mean curvature surface}

From 1956 to 1958, Alexandrov proved an unexpected theorem: an embedded surface (i.e. the surface does not intersect with itself) with constant mean curvature in $\mathbb{E}^{3}$ must be a spherical surface [23]. Thus the closed bilayer with constant mean curvature must be a sphere. For a sphere with radius $R$, we have $H=-1 / R$ and $K=1 / R^{2}$. Substituting them into (3), we arrive at

$$
p R^{2}+2 \mu R-k_{c} c_{0}\left(2-c_{0} R\right)=0 .
$$

This equation gives the spherical radius under the osmotic pressure $p$.

\subsubsection{Biconcave discoid shape and $\sqrt{2}$ torus It is instructive to find some} axisymmetrical solutions to the shape equation (33). To do that, we denote $\mathbf{r}=$ $\{u \cos v, u \sin v, z\}, \psi=\arctan \left[\frac{d z(u)}{d u}\right]$, and $\Psi=\sin \psi$. Thus (3) is transformed into

$$
\begin{aligned}
& \left(\Psi^{2}-1\right) \frac{d^{3} \Psi}{d u^{3}}+\Psi \frac{d^{2} \Psi}{d u^{2}} \frac{d \Psi}{d u}-\frac{1}{2}\left(\frac{d \Psi}{d u}\right)^{3}-\frac{p}{k_{c}} \\
+ & \frac{2\left(\Psi^{2}-1\right)}{u} \frac{d^{2} \Psi}{d u^{2}}+\frac{3 \Psi}{2 u}\left(\frac{d \Psi}{d u}\right)^{2}+\left(\frac{c_{0}^{2}}{2}+\frac{2 c_{0} \Psi}{u}+\frac{\mu}{k_{c}}-\frac{3 \Psi^{2}-2}{2 u^{2}}\right) \frac{d \Psi}{d u} \\
+ & \left(\frac{c_{0}^{2}}{2}+\frac{\mu}{k_{c}}-\frac{1}{u^{2}}\right) \frac{\Psi}{u}+\frac{\Psi^{3}}{2 u^{3}}=0 .
\end{aligned}
$$

To find the solution of (63) that satisfies $\Psi=0$ when $u=0$, we consider the asymptotic form of (63) at $u=0$ :

$$
\frac{d^{3} \Psi}{d u^{3}}+\frac{2}{u} \frac{d^{2} \Psi}{d u^{2}}-\frac{1}{u^{2}} \frac{d \Psi}{d u}+\frac{\Psi}{u^{3}}=0
$$

Please notice that there are two misprints in our previous paper [13]. Above equation is the Euler differential equation and has the general solution $\Psi=\alpha_{1} / u+\alpha_{2} u+\alpha_{3} u \ln u$ with three integral constants $\alpha_{1}=0, \alpha_{2}$, and $\alpha_{3}$. The asymptotic solution hints that $\Psi=-c_{0} u \ln \left(u / u_{B}\right)$ might be a solution to (63) which requires $p=\mu=0$. When $0<c_{0} u_{B}<e, \Psi=-c_{0} u \ln \left(u / u_{B}\right)$ corresponds to the biconcave discoid shape [6,24].

Otherwise, when $\mu / k_{c}=-2 \alpha c_{0}-c_{0}^{2} / 2$ and $p / k_{c}=-2 \alpha^{2} c_{0}, \Psi=\alpha u+\sqrt{2}$ satisfies (63). This solution corresponds to a torus with the ratio of its two radii being exactly $\sqrt{2}$ if $\alpha<0[6,25]$.

\subsection{Mechanical stability of spherical bilayers}

A spherical bilayer can be described by $\mathbf{r}=R(\sin \theta \cos \phi, \sin \theta \sin \phi, \cos \theta)$ with $R$ satisfying (62). We have $H=-1 / R, K=1 / R^{2}, \tilde{\nabla}=-(1 / R) \nabla, \nabla \cdot \tilde{\nabla}=-(1 / R) \nabla^{2}$ and $\nabla^{2}=\frac{1}{R^{2} \sin \theta} \frac{\partial}{\partial \theta}\left(\sin \theta \frac{\partial}{\partial \theta}\right)+\frac{1}{R^{2} \sin ^{2} \theta} \frac{\partial^{2}}{\partial \phi^{2}}$. If we take $\mathcal{E}_{H}=\left(k_{c} / 2\right)\left(2 H+c_{0}\right)^{2}+\mu$, (61) is transformed into

$$
\delta^{2} \mathcal{F}=\left(2 c_{0} k_{c} / R+p R\right) \int_{0}^{\pi} \sin \theta d \theta \int_{0}^{2 \pi} d \phi \Omega_{3}^{2}
$$


Geometric theory on the elasticity of bio-membranes

$$
\begin{aligned}
& +\left(k_{c} c_{0} R+2 k_{c}+p R^{3} / 2\right) \int_{0}^{\pi} \sin \theta d \theta \int_{0}^{2 \pi} d \phi \Omega_{3} \nabla^{2} \Omega_{3} \\
& +k_{c} R^{2} \int_{0}^{\pi} \sin \theta d \theta \int_{0}^{2 \pi} d \phi\left(\nabla^{2} \Omega_{3}\right)^{2} .
\end{aligned}
$$

Expand $\Omega_{3}$ with the spherical harmonic functions [26]:

$$
\Omega_{3}=\sum_{l=0}^{\infty} \sum_{m=-l}^{m=l} a_{l m} Y_{l m}(\theta, \phi), \quad a_{l m}^{*}=(-1)^{m} a_{l,-m} .
$$

If considering $\nabla^{2} Y_{l m}=-l(l+1) Y_{l m} / R^{2}$ and $\int_{0}^{\pi} \sin \theta d \theta \int_{0}^{2 \pi} d \phi Y_{l m}^{*} Y_{l^{\prime} m^{\prime}}=\delta_{m m^{\prime}} \delta_{l l^{\prime}}$, we transform (65) into

$$
\delta^{2} \mathcal{F}=(R / 2) \sum_{l, m}\left|a_{l m}\right|^{2}[l(l+1)-2]\left\{2 k_{c} / R^{3}\left[l(l+1)-c_{0} R\right]-p\right\} .
$$

Denote that

$$
p_{l}=\left(2 k_{c} / R^{3}\right)\left[l(l+1)-c_{0} R\right] \quad(l=2,3, \cdots) .
$$

When $p>p_{l}, \delta^{2} \mathcal{F}$ can take negative value. Therefore, we can take the critical pressure as

$$
p_{c}=\min \left\{p_{l}\right\}=p_{2}=\left(2 k_{c} / R^{3}\right)\left(6-c_{0} R\right) .
$$

In this case, the spherical bilayer will be inclined to transform into the biconcave discoid shape.

\section{Open lipid bilayers}

In this section, we will deal with the variational problems on surface $M$ with edge $C$ as shown in Fig 1, and discuss the shape equation and boundary conditions of open lipid bilayers with free edges.

\subsection{First order variational problems on an open surface}

In this subsection, we will discuss the first order variation of the functional

$$
\mathcal{F}=\int_{M} \mathcal{E}(2 H[\mathbf{r}], K[\mathbf{r}]) d A+\int_{C} \Gamma\left(k_{n}, k_{g}\right) d s .
$$

Denote $\mathcal{F}_{e}=\int_{M} \mathcal{E}(2 H[\mathbf{r}], K[\mathbf{r}]) d A$, and $\mathcal{F}_{C}=\int_{C} \Gamma\left(k_{n}, k_{g}\right) d s$.

In terms of Appendix B, we have $\omega_{2}=0, d s=\omega_{1}, k_{n}=a, k_{g} d s=\omega_{12}, \tau_{g}=b$ in curve $C$. Using (27) $\sim(36)$, we can arrive at

$$
\begin{array}{rl}
\delta_{1} \mathcal{F}_{C}=\int_{C} & d\left(\Gamma \Omega_{1}\right)=0 \\
\delta_{2} \mathcal{F}_{C}=\int_{C} & {\left[\frac{d^{2}}{d s^{2}}\left(\frac{\partial \Gamma}{\partial k_{g}}\right)+K \frac{\partial \Gamma}{\partial k_{g}}-k_{g}\left(\Gamma-\frac{\partial \Gamma}{\partial k_{g}} k_{g}\right)\right.} \\
& \left.+2\left(k_{n}-H\right) k_{g} \frac{\partial \Gamma}{\partial k_{n}}-\tau_{g} \frac{d}{d s}\left(\frac{\partial \Gamma}{\partial k_{n}}\right)-\frac{d}{d s}\left(\tau_{g} \frac{\partial \Gamma}{\partial k_{n}}\right)\right] \Omega_{2} d s
\end{array}
$$


Geometric theory on the elasticity of bio-membranes

$$
\begin{aligned}
\delta_{3} \mathcal{F}_{C}=\int_{C} & {\left[\frac{d^{2}}{d s^{2}}\left(\frac{\partial \Gamma}{\partial k_{n}}\right)+\frac{\partial \Gamma}{\partial k_{n}}\left(k_{n}^{2}-\tau_{g}^{2}\right)+\tau_{g} \frac{d}{d s}\left(\frac{\partial \Gamma}{\partial k_{g}}\right)\right.} \\
+ & \left.\frac{d}{d s}\left(\tau_{g} \frac{\partial \Gamma}{\partial k_{g}}\right)-\left(\Gamma-\frac{\partial \Gamma}{\partial k_{g}} k_{g}\right) k_{n}\right] \Omega_{3} d s \\
+ & \int_{C}\left(\frac{\partial \Gamma}{\partial k_{g}} k_{n}-\frac{\partial \Gamma}{\partial k_{n}} k_{g}\right) \Omega_{323} d s .
\end{aligned}
$$

In particular, (39), (49) and (50) are still applicable. Consequently,

$$
\begin{aligned}
\delta_{1} \mathcal{F}_{e}= & \int_{M} d\left(\mathcal{E} \omega_{2} \Omega_{1}\right)=\int_{C} \mathcal{E} \omega_{2} \Omega_{1}=0 \\
\delta_{2} \mathcal{F}_{e}= & -\int_{M} d\left(\mathcal{E} \omega_{1} \Omega_{2}\right)=-\int_{C} \mathcal{E} \Omega_{2} d s \\
\delta_{3} \mathcal{F}_{e}= & \int_{M}\left[\left(\nabla^{2}+4 H^{2}-2 K\right) \frac{\partial \mathcal{E}}{\partial(2 H)}+(\nabla \cdot \tilde{\nabla}+2 K H) \frac{\partial \mathcal{E}}{\partial K}-2 H \mathcal{E}\right] \Omega_{3} d A \\
& +\int_{C}\left[\mathbf{e}_{2} \cdot \nabla\left[\frac{\partial \mathcal{E}}{\partial(2 H)}\right]+\mathbf{e}_{2} \cdot \tilde{\nabla}\left(\frac{\partial \mathcal{E}}{\partial K}\right)-\frac{d}{d s}\left(\frac{\partial \mathcal{E}}{\partial K}\right)\right] \Omega_{3} d s \\
& +\int_{C}\left[-\frac{\partial \mathcal{E}}{\partial(2 H)}-k_{n} \frac{\partial \mathcal{E}}{\partial K}\right] \Omega_{323} d s .
\end{aligned}
$$

The functions $\Omega_{323}, \Omega_{2}$, and $\Omega_{3}$ can be regarded as virtual displacements. Thus $\delta \mathcal{F}=\left(\delta_{1}+\delta_{2}+\delta_{3}\right)\left(\mathcal{F}_{e}+\mathcal{F}_{C}\right)=0$ gives

$$
\begin{aligned}
& \left(\nabla^{2}+4 H^{2}-2 K\right) \frac{\partial \mathcal{E}}{\partial(2 H)}+(\nabla \cdot \tilde{\nabla}+2 K H) \frac{\partial \mathcal{E}}{\partial K}-2 H \mathcal{E}=0, \\
& \mathbf{e}_{2} \cdot \nabla\left[\frac{\partial \mathcal{E}}{\partial(2 H)}\right]+\mathbf{e}_{2} \cdot \tilde{\nabla}\left(\frac{\partial \mathcal{E}}{\partial K}\right)-\frac{d}{d s}\left(\tau_{g} \frac{\partial \mathcal{E}}{\partial K}\right)+\frac{d^{2}}{d s^{2}}\left(\frac{\partial \Gamma}{\partial k_{n}}\right)+\frac{\partial \Gamma}{\partial k_{n}}\left(k_{n}^{2}-\tau_{g}^{2}\right) \\
& \quad+\tau_{g} \frac{d}{d s}\left(\frac{\partial \Gamma}{\partial k_{g}}\right)+\frac{d}{d s}\left(\tau_{g} \frac{\partial \Gamma}{\partial k_{g}}\right)-\left.\left(\Gamma-\frac{\partial \Gamma}{\partial k_{g}} k_{g}\right) k_{n}\right|_{C}=0, \\
& -\frac{\partial \mathcal{E}}{\partial(2 H)}-k_{n} \frac{\partial \mathcal{E}}{\partial K}+\frac{\partial \Gamma}{\partial k_{g}} k_{n}-\left.\frac{\partial \Gamma}{\partial k_{n}} k_{g}\right|_{C}=0, \\
& \frac{d^{2}}{d s^{2}}\left(\frac{\partial \Gamma}{\partial k_{g}}\right)+K \frac{\partial \Gamma}{\partial k_{g}}-k_{g}\left(\Gamma-\frac{\partial \Gamma}{\partial k_{g}} k_{g}\right)+2\left(k_{n}-H\right) k_{g} \frac{\partial \Gamma}{\partial k_{n}} \\
& -\tau_{g} \frac{d}{d s}\left(\frac{\partial \Gamma}{\partial k_{n}}\right)-\frac{d}{d s}\left(\tau_{g} \frac{\partial \Gamma}{\partial k_{n}}\right)-\left.\mathcal{E}\right|_{C}=0 .
\end{aligned}
$$

Among above equations, (77) determines the shape of the surface $M$, and (78) $\sim(80)$ determine the position of curve $C$ in the surface $M$.

\subsection{Shape equation and boundary conditions of open lipid bilayers}

In order to obtain the shape equation and boundary conditions of an open lipid bilayer with an edge $C$, we take $\mathcal{E}=\left(k_{c} / 2\right)\left(2 H+c_{0}\right)^{2}+\bar{k} K+\mu$ and $\Gamma=\frac{1}{2} k_{b}\left(k_{n}^{2}+k_{g}^{2}\right)+\gamma$ with 
$k_{b}$ and $\gamma$ being constants. In this case, (77) (80) are transformed into

$$
\begin{aligned}
& k_{c}\left(2 H+c_{0}\right)\left(2 H^{2}-c_{0} H-2 K\right)+k_{c} \nabla^{2}(2 H)-2 \mu H=0, \\
& k_{b}\left[d^{2} k_{n} / d s^{2}+k_{n}\left(\kappa^{2} / 2-\tau_{g}^{2}\right)+\tau_{g} d k_{g} / d s+d\left(\tau_{g} k_{g}\right) / d s\right] \\
& \quad+k_{c} \mathbf{e}_{2} \cdot \nabla(2 H)-\bar{k} d \tau_{g} / d s-\left.\gamma k_{n}\right|_{C}=0, \\
& k_{c}\left(2 H+c_{0}\right)+\left.\bar{k} k_{n}\right|_{C}=0, \\
& k_{b}\left[d^{2} k_{g} / d s^{2}+k_{g}\left(\kappa^{2} / 2-\tau_{g}^{2}\right)-\tau_{g} d k_{n} / d s-d\left(\tau_{g} k_{n}\right) / d s\right] \\
& \quad-\left.\left[\left(k_{c} / 2\right)\left(2 H+c_{0}\right)^{2}+\bar{k} K+\mu+\gamma k_{g}\right]\right|_{C}=0,
\end{aligned}
$$

where $\kappa^{2}=k_{n}^{2}+k_{g}^{2}$.

In fact, the above four equations express the force and moment equilibrium equations of the surface and the edge: (181) represents the force equilibrium equation of point in the surface $M$ along $\mathbf{e}_{3}$ direction; (82) represents the force equilibrium equation of point in the curve $C$ along $\mathbf{e}_{3}$ direction; (83) represents the bending moment equilibrium equation of point in the curve $C$ around $\mathbf{e}_{1}$ direction; (84) represents the force equilibrium equation of point in the curve $C$ along $\mathbf{e}_{2}$ direction.

If $k_{b}=0$, (81) and (83) remain unchanged, but (82) and (84) are simplified to

$$
\begin{aligned}
& k_{c} \mathbf{e}_{2} \cdot \nabla(2 H)-\bar{k} d \tau_{g} / d s-\left.\gamma k_{n}\right|_{C}=0, \\
& \left(k_{c} / 2\right)\left(2 H+c_{0}\right)^{2}+\bar{k} K+\mu+\left.\gamma k_{g}\right|_{C}=0 .
\end{aligned}
$$

\subsection{Two-component lipid bilayer}

In this subsection, we study a closed bilayer consists of two domains containing different kinds of lipid. This problem in axisymmetrical case was theoretically discussed by Jülicher and Lipowsky [27]. The shapes of two-component bilayers also were observed in recent experiment [28].

We assume that the boundary between two domains is a smooth curve and the bilayer is still a smooth surface. The free energy is written as

$$
\begin{aligned}
\mathcal{F}= & p \int_{V} d V+\int_{M_{I}}\left[\left(k_{c}^{I} / 2\right)\left(2 H+c_{0}^{I}\right)^{2}+\bar{k}^{I} K+\mu^{I}\right] d A \\
& +\int_{M_{I I}}\left[\left(k_{c}^{I I} / 2\right)\left(2 H+c_{0}^{I I}\right)^{2}+\bar{k}^{I I} K+\mu^{I I}\right] d A+\gamma \int_{C} d s .
\end{aligned}
$$

In terms of the discussions on closed bilayers in section 3 and above discussions in this section, we can promptly write the shape equations of the two-component bilayer without any symmetrical assumption as

$$
p-2 \mu^{i} H+k_{c}^{i}\left(2 H+c_{0}\right)\left(2 H^{2}-c_{0}^{i} H-2 K\right)+k_{c}^{i} \nabla^{2}(2 H)=0,
$$

where the superscripts $i=I$ and $I I$ represent the two lipid domains, respectively. And the boundary conditions are as follows:

$$
\begin{aligned}
& k_{c}^{I} \mathbf{e}_{2} \cdot \nabla(2 H)-\bar{k}^{I} d \tau_{g} / d s+k_{c}^{I I} \mathbf{e}_{2} \cdot \nabla(2 H)-\bar{k}^{I I} d \tau_{g} / d s-\left.\gamma k_{n}\right|_{C}=0, \\
& k_{c}^{I}\left(2 H+c_{0}^{I}\right)+\bar{k}^{I} k_{n}-\left.\left[k_{c}^{I I}\left(2 H+c_{0}^{I I}\right)+\bar{k}^{I I} k_{n}\right]\right|_{C}=0, \\
& \left(k_{c}^{I} / 2\right)\left(2 H+c_{0}^{I}\right)^{2}+\bar{k}^{I} K+\mu^{I}
\end{aligned}
$$


Geometric theory on the elasticity of bio-membranes

$$
-\left[\left(k_{c}^{I I} / 2\right)\left(2 H+c_{0}^{I I}\right)^{2}+\bar{k}^{I I} K+\mu^{I I}\right]+\left.\gamma k_{g}\right|_{C}=0 .
$$

In above equations, the positive direction of curve $C$ is set to along $\mathbf{e}_{1}$ of the lipid

domain consisting of component $I$. Furthermore, (88) (91) are also applied to describe the closed bilayer with more than two domains. But the boundary conditions are not applied to the bilayer with a sharp angle across the boundary between domains.

\section{Cell membranes with cross-linking structures}

Cell membranes contain cross-linking protein structures. As is well known, rubber also consists of cross-linking polymer structures [29]. In this section, we first drive the free energy of cell membrane with cross-linking protein structure by analogy with the rubber elasticity. Secondly, we derive the shape equation and in-plane stain equations of cell membrane by taking the first order variation of the free energy. Lastly, we discuss the mechanical stability of spherical cell membrane.

\subsection{The free energy of cell membrane}

Above all, we discuss the free energy change of a Gaussian chain in a small strain field

$$
\epsilon=\left(\begin{array}{ccc}
\varepsilon_{x x} & \varepsilon_{x y} & 0 \\
\varepsilon_{x y} & \varepsilon_{y y} & 0 \\
0 & 0 & \varepsilon_{z z}
\end{array}\right)
$$

with $\varepsilon_{z z}=-\left(\varepsilon_{x x}+\varepsilon_{x x}\right)$ expressed in an orthogonal coordinate system Oxyz.

Assume that one end of the chain is fixed at origin $O$ while another is denoted by $\mathbf{R}_{N}$ before undergoing the strains, where $N$ is the number of the segments of the chain. The partition function of the chain can be calculated by path integrals [30]:

$$
Z=\int_{\mathbf{R}_{0}}^{\mathbf{R}_{N}} D\left[\mathbf{R}_{n}\right] \exp \left[-\frac{3}{2 L^{2}} \int_{0}^{N} d n\left(\frac{\partial \mathbf{R}_{n}}{\partial n}\right)^{2}\right]=\sigma \exp \left[-\frac{3\left(\mathbf{R}_{N}-\mathbf{R}_{0}\right)^{2}}{2 N L^{2}}\right],
$$

where $\sigma$ is a constant, and $L$ is the segment length. After undergoing the strains, the partition function is changed to

$$
Z_{\epsilon}=\sigma \exp \left[-\frac{3\left(\mathbf{R}_{N}-\mathbf{R}_{0}\right)_{\epsilon}^{2}}{2 N L^{2}}\right] .
$$

Considering the relation $\left(\mathbf{R}_{N}-\mathbf{R}_{0}\right)_{\epsilon}^{2}=\left[(1+\epsilon) \cdot\left(\mathbf{R}_{N}-\mathbf{R}_{0}\right)\right]^{2}$ and the distribution function of end-to-end distance $P\left(\mathbf{R}_{N}-\mathbf{R}_{0}\right)=\frac{Z}{\int d \mathbf{R}_{N} Z}$, we can obtain the free energy change as a result of the strains:

$$
\begin{aligned}
f_{s} & =-k_{B} T \int d \mathbf{R}_{N}\left(\ln Z_{\epsilon}-\ln Z\right) P\left(\mathbf{R}_{N}-\mathbf{R}_{0}\right) \\
& =k_{B} T\left[\left(\varepsilon_{x x}+\varepsilon_{y y}\right)^{2}-\left(\varepsilon_{x x} \varepsilon_{y y}-\varepsilon_{x y}^{2}\right)\right] .
\end{aligned}
$$

For the cell membrane with the cross-linking structure, we assume that: (i) The membrane is a smooth surface and junction points between protein chains are confined in the vicinity of the surface and freely depart from it within the range of $\pm h / 2$, where $h$ is 
the thickness of the membrane. (ii) There is no change of volume occupied by the crosslinking structure on deformation. On average, there are $\mathcal{N}$ protein chains per volume. (iii) The protein chain can be regarded as a Gaussian chain with the mean end-to-end distance much smaller than the dimension of the cell membrane. (iv) The junction points move on deformation as if they were embedded in an elastic continuum (Affine deformation assumption). (v) The free energy of the cell membrane on deformation is the sum of the free energies of closed lipid bilayer and the cross-linking structure. The free energy of cross-linking structure is the sum of the free energies of individual protein chains.

If the cell membrane undergoing the small in-plane deformation $\left(\begin{array}{ll}\varepsilon_{11} & \varepsilon_{12} \\ \varepsilon_{12} & \varepsilon_{22}\end{array}\right)$, where "1" and "2" represent two orthogonal directions of the membrane surface, we can obtain the free energy of a protein chain on deformation with the similar form of (93) under above assumptions (i) (iv). Using above assumption (v), we have the free energy of the cell membrane under the osmotic pressure $p$ :

$$
\mathcal{F}=\int_{M}\left(\mathcal{E}_{d}+\mathcal{E}_{H}\right) d A+p \int_{V} d V
$$

where $\mathcal{E}_{H}=\left(k_{c} / 2\right)\left(2 H+c_{0}\right)^{2}+\mu$ and $\mathcal{E}_{d}=\left(k_{d} / 2\right)\left[(2 J)^{2}-Q\right]$ with $k_{d}=2 \mathcal{N} h k_{B} T$, $2 J=\varepsilon_{11}+\varepsilon_{22}, Q=\varepsilon_{11} \varepsilon_{22}-\varepsilon_{12}^{2}$.

Remark 5.1 We do not write the term of $\bar{k} K$ in (94) because $\int_{M} \bar{k} K d A$ is a constant for closed surface (see Appendix C).

\subsection{Strain analysis}

If a point $\mathbf{r}_{0}$ in a surface undergoing a displacement $\mathbf{u}$ to arrive at point $\mathbf{r}$, we have $d \mathbf{u}=d \mathbf{r}-d \mathbf{r}_{0}$ and $\delta_{i} d \mathbf{u}=\delta_{i} d \mathbf{r}(i=1,2,3)$.

If denote $d \mathbf{r}=\omega_{1} \mathbf{e}_{1}+\omega_{2} \mathbf{e}_{2}$ and $d \mathbf{u}=\mathbf{U}_{1} \omega_{1}+\mathbf{U}_{2} \omega_{2}$ with $\left|\mathbf{U}_{1}\right| \ll 1,\left|\mathbf{U}_{2}\right| \ll 1$, we can define the strains [31]:

$$
\begin{aligned}
& \varepsilon_{11}=\left[\frac{d \mathbf{u} \cdot \mathbf{e}_{1}}{\left|d \mathbf{r}_{0}\right|}\right]_{\omega_{2}=0} \approx \mathbf{U}_{1} \cdot \mathbf{e}_{1}, \\
& \varepsilon_{22}=\left[\frac{d \mathbf{u} \cdot \mathbf{e}_{2}}{\left|d \mathbf{r}_{0}\right|}\right]_{\omega_{1}=0} \approx \mathbf{U}_{2} \cdot \mathbf{e}_{2}, \\
& \varepsilon_{12}=\frac{1}{2}\left[\left(\frac{d \mathbf{u} \cdot \mathbf{e}_{2}}{\left|d \mathbf{r}_{0}\right|}\right)_{\omega_{2}=0}+\left(\frac{d \mathbf{u} \cdot \mathbf{e}_{1}}{\left|d \mathbf{r}_{0}\right|}\right)_{\omega_{1}=0}\right] \approx \frac{1}{2}\left(\mathbf{U}_{1} \cdot \mathbf{e}_{2}+\mathbf{U}_{2} \cdot \mathbf{e}_{1}\right) .
\end{aligned}
$$

Using $\delta_{i} d \mathbf{u}=\delta_{i} d \mathbf{r}$ and the definitions of strains (95) (97), we can obtain the variational relations:

$$
\begin{aligned}
\delta_{i} \varepsilon_{11} \omega_{1} \wedge \omega_{2}= & \left(1-\varepsilon_{11}\right) \delta_{i} \omega_{1} \wedge \omega_{2}-\mathbf{U}_{2} \cdot \mathbf{e}_{1} \delta_{i} \omega_{2} \wedge \omega_{2} \\
& +\Omega_{i 12} \mathbf{U}_{1} \cdot \mathbf{e}_{2} \omega_{1} \wedge \omega_{2}+\Omega_{i 13} \mathbf{U}_{1} \cdot \mathbf{e}_{3} \omega_{1} \wedge \omega_{2}, \\
\delta_{i} \varepsilon_{12} \omega_{1} \wedge \omega_{2}= & \frac{1}{2}\left[\left(1-\varepsilon_{11}\right) \omega_{1} \wedge \delta_{i} \omega_{1}+\left(1-\varepsilon_{22}\right) \delta_{i} \omega_{2} \wedge \omega_{2}-\mathbf{U}_{2} \cdot \mathbf{e}_{1} \omega_{1} \wedge \delta_{i} \omega_{2}\right.
\end{aligned}
$$


Geometric theory on the elasticity of bio-membranes

$$
\begin{aligned}
& -\mathbf{U}_{1} \cdot \mathbf{e}_{2} \delta_{i} \omega_{1} \wedge \omega_{2}+\Omega_{i 21}\left(\varepsilon_{11}-\varepsilon_{22}\right) \omega_{1} \wedge \omega_{2} \\
& \left.+\Omega_{i 23} \mathbf{U}_{1} \cdot \mathbf{e}_{3} \omega_{1} \wedge \omega_{2}+\Omega_{i 13} \mathbf{U}_{2} \cdot \mathbf{e}_{3} \omega_{1} \wedge \omega_{2}\right] \\
\delta_{i} \varepsilon_{22} \omega_{1} \wedge \omega_{2}= & \left(1-\varepsilon_{22}\right) \omega_{1} \wedge \delta_{i} \omega_{2}-\mathbf{U}_{1} \cdot \mathbf{e}_{2} \omega_{1} \wedge \delta_{i} \omega_{1} \\
& +\Omega_{i 21} \mathbf{U}_{2} \cdot \mathbf{e}_{1} \omega_{1} \wedge \omega_{2}+\Omega_{i 23} \mathbf{U}_{2} \cdot \mathbf{e}_{3} \omega_{1} \wedge \omega_{2} .
\end{aligned}
$$

The leading terms of above relations are:

$$
\begin{aligned}
& \delta_{i} \varepsilon_{11} \omega_{1} \wedge \omega_{2}=\delta_{i} \omega_{1} \wedge \omega_{2}, \\
& \delta_{i} \varepsilon_{12} \omega_{1} \wedge \omega_{2}=\frac{1}{2}\left[\omega_{1} \wedge \delta_{i} \omega_{1}+\delta_{i} \omega_{2} \wedge \omega_{2}\right], \\
& \delta_{i} \varepsilon_{22} \omega_{1} \wedge \omega_{2}=\omega_{1} \wedge \delta_{i} \omega_{2} .
\end{aligned}
$$

Thus,

$$
\begin{aligned}
\delta_{i}(2 J) \omega_{1} \wedge \omega_{2} & =\delta_{i}\left(\varepsilon_{11}+\varepsilon_{22}\right) \omega_{1} \wedge \omega_{2}=\delta_{i} \omega_{1} \wedge \omega_{2}+\omega_{1} \wedge \delta_{i} \omega_{2}, \\
\delta_{i} Q \omega_{1} \wedge \omega_{2} & =\delta_{i}\left(\varepsilon_{11} \varepsilon_{22}-\varepsilon_{12}^{2}\right) \omega_{1} \wedge \omega_{2} \\
& =\left(\varepsilon_{11} \omega_{1}+\varepsilon_{12} \omega_{2}\right) \wedge \delta_{i} \omega_{2}-\left(\varepsilon_{12} \omega_{1}+\varepsilon_{22} \omega_{2}\right) \wedge \delta_{i} \omega_{1} .
\end{aligned}
$$

Considering equations (27) (35), (101) and (102), we have

$$
\begin{aligned}
& \delta_{1}(2 J) \omega_{1} \wedge \omega_{2}=d\left(\Omega_{1} \omega_{2}\right), \\
& \delta_{1} Q \omega_{1} \wedge \omega_{2}=\left(\varepsilon_{11} d \omega_{2}-\varepsilon_{12} d \omega_{1}\right) \Omega_{1}-\left(\varepsilon_{12} \omega_{1}+\varepsilon_{22} \omega_{2}\right) \wedge d \Omega_{1} ; \\
& \delta_{2}(2 J) \omega_{1} \wedge \omega_{2}=-d\left(\Omega_{2} \omega_{1}\right), \\
& \delta_{2} Q \omega_{1} \wedge \omega_{2}=\left(\varepsilon_{11} \omega_{1}+\varepsilon_{12} \omega_{2}\right) \wedge d \Omega_{2}+\Omega_{2}\left(\varepsilon_{12} d \omega_{2}-\varepsilon_{22} d \omega_{1}\right) ; \\
& \delta_{3}(2 J) \omega_{1} \wedge \omega_{2}=-2 H \Omega_{3} d A, \\
& \delta_{3} Q \omega_{1} \wedge \omega_{2}=\left[-2 H(2 J)+a \varepsilon_{11}+2 b \varepsilon_{12}+c \varepsilon_{22}\right] \Omega_{3} d A .
\end{aligned}
$$

\subsection{Shape equation and in-plane strain equations of cell membranes}

To obtain the shape equation and in-plane strain equations of cell membranes, we must take the first order variation of the functional (94). Denote $\mathcal{F}_{d}=\int_{M} \mathcal{E}_{d} d A$ and $\mathcal{F}_{c p}=\int_{M} \mathcal{E}_{H} d A+p \int_{V} d V$.

From (103) (108), we can calculate that:

$$
\begin{aligned}
\delta_{1} \mathcal{F}_{d}= & \int_{M}\left[\frac{\partial \mathcal{E}_{d}}{\partial(2 J)} \delta_{1}(2 J) d A+\frac{\partial \mathcal{E}_{d}}{\partial Q} \delta_{1} Q d A+\mathcal{E}_{d}(2 J, Q) \delta_{1} d A\right] \\
= & -\int_{M} d\left[\frac{\partial \mathcal{E}_{d}}{\partial(2 J)}+\mathcal{E}_{d}(2 J, Q)\right] \wedge \omega_{2} \Omega_{1}+\int_{M} \frac{\partial \mathcal{E}_{d}}{\partial Q}\left(\varepsilon_{11} d \omega_{2}-\varepsilon_{12} d \omega_{1}\right) \Omega_{1} \\
& -\int_{M} \Omega_{1} d\left[\left(\varepsilon_{12} \omega_{1}+\varepsilon_{22} \omega_{2}\right) \frac{\partial \mathcal{E}_{d}}{\partial Q}\right], \\
\delta_{2} \mathcal{F}_{d}= & \int_{M}\left[\frac{\partial \mathcal{E}_{d}}{\partial(2 J)} \delta_{2}(2 J) d A+\frac{\partial \mathcal{E}_{d}}{\partial Q} \delta_{2} Q d A+\mathcal{E}_{d}(2 J, Q) \delta_{2} d A\right] \\
= & \int_{M} d\left[\frac{\partial \mathcal{E}_{d}}{\partial(2 J)}+\mathcal{E}_{d}(2 J, Q)\right] \wedge \omega_{1} \Omega_{2}+\int_{M} \frac{\partial \mathcal{E}_{d}}{\partial Q}\left(\varepsilon_{12} d \omega_{2}-\varepsilon_{22} d \omega_{1}\right) \Omega_{2} \\
& +\int_{M} \Omega_{2} d\left[\frac{\partial \mathcal{E}_{d}}{\partial Q}\left[\left(\varepsilon_{11} \omega_{1}+\varepsilon_{12} \omega_{2}\right)\right]\right.
\end{aligned}
$$


Geometric theory on the elasticity of bio-membranes

$$
\begin{aligned}
\delta_{3} \mathcal{F}_{d}= & \int_{M}\left[\frac{\partial \mathcal{E}_{d}}{\partial(2 J)} \delta_{3}(2 J) d A+\frac{\partial \mathcal{E}_{d}}{\partial Q} \delta_{3} Q d A+\mathcal{E}_{d}(2 J, Q) \delta_{3} d A\right] \\
= & \int_{M} \frac{\partial \mathcal{E}_{d}}{\partial Q}\left[a \varepsilon_{11}+2 b \varepsilon_{12}+c \varepsilon_{22}\right] \Omega_{3} d A \\
& -\int_{M} 2 H\left[\frac{\partial \mathcal{E}_{d}}{\partial(2 J)}+\mathcal{E}_{d}+(2 J) \frac{\partial \mathcal{E}_{d}}{\partial Q}\right] \Omega_{3} d A .
\end{aligned}
$$

Otherwise, section 3 tells us:

$$
\begin{aligned}
\delta_{1} \mathcal{F}_{c p} & =\delta_{2} \mathcal{F}_{c p}=0, \\
\delta_{3} \mathcal{F}_{c p} & =\int_{M}\left[\left(\nabla^{2}+4 H^{2}-2 K\right) \frac{\partial \mathcal{E}_{H}}{\partial(2 H)}-2 H \mathcal{E}_{H}+p\right] \Omega_{3} d A .
\end{aligned}
$$

Therefore, $\delta_{i} \mathcal{F}=\delta_{i} \mathcal{F}_{d}+\delta_{i} \mathcal{F}_{c p}=0$ gives:

$$
\begin{aligned}
& -d\left[\frac{\partial \mathcal{E}_{d}}{\partial(2 J)}+\mathcal{E}_{d}\right] \wedge \omega_{2}+\frac{\partial \mathcal{E}_{d}}{\partial Q}\left(\varepsilon_{11} d \omega_{2}-\varepsilon_{12} d \omega_{1}\right) \\
& \quad-d\left[\left(\varepsilon_{12} \omega_{1}+\varepsilon_{22} \omega_{2}\right) \frac{\partial \mathcal{E}_{d}}{\partial Q}\right]=0 \\
& d\left[\frac{\partial \mathcal{E}_{d}}{\partial(2 J)}+\mathcal{E}_{d}\right] \wedge \omega_{1}+\frac{\partial \mathcal{E}_{d}}{\partial Q}\left(\varepsilon_{12} d \omega_{2}-\varepsilon_{22} d \omega_{1}\right) \\
& \quad+d\left[\frac{\partial \mathcal{E}_{d}}{\partial Q}\left(\varepsilon_{11} \omega_{1}+\varepsilon_{12} \omega_{2}\right)\right]=0, \\
& \left(\nabla^{2}+4 H^{2}-2 K\right) \frac{\partial \mathcal{E}_{H}}{\partial(2 H)}-2 H\left[\mathcal{E}_{H}+\frac{\partial \mathcal{E}_{d}}{\partial(2 J)}+\mathcal{E}_{d}+(2 J) \frac{\partial \mathcal{E}_{d}}{\partial Q}\right] \\
& +p+\frac{\partial \mathcal{E}_{d}}{\partial Q}\left[a \varepsilon_{11}+2 b \varepsilon_{12}+c \varepsilon_{22}\right]=0 .
\end{aligned}
$$

Substituting $\mathcal{E}_{H}=\frac{k_{c}}{2}\left(2 H+c_{0}\right)^{2}+\mu$ and $\mathcal{E}_{d}=\frac{k_{d}}{2}\left[(2 J)^{2}-Q\right]$ into above three equations, we obtain:

$$
\begin{aligned}
& k_{d}\left[-d(2 J) \wedge \omega_{2}-\frac{1}{2}\left(\varepsilon_{11} d \omega_{2}-\varepsilon_{12} d \omega_{1}\right)+\frac{1}{2} d\left(\varepsilon_{12} \omega_{1}+\varepsilon_{22} \omega_{2}\right)\right]=0, \\
& k_{d}\left[d(2 J) \wedge \omega_{1}-\frac{1}{2}\left(\varepsilon_{12} d \omega_{2}-\varepsilon_{22} d \omega_{1}\right)-\frac{1}{2} d\left(\varepsilon_{11} \omega_{1}+\varepsilon_{12} \omega_{2}\right)\right]=0, \\
& p-2 H\left(\mu+k_{d} J\right)+k_{c}\left(2 H+c_{0}\right)\left(2 H^{2}-c_{0} H-2 K\right)+k_{c} \nabla^{2}(2 H) \\
& \quad-\frac{k_{d}}{2}\left(a \varepsilon_{11}+2 b \varepsilon_{12}+c \varepsilon_{22}\right)=0 .
\end{aligned}
$$

(115) and (116) are called in-plane strain equations of the cell membrane, while (117) is the shape equation.

Remark 5.2 The higher order terms of $\varepsilon_{i j}(i, j=1,2)$ are neglected in above three equations.

Obviously, if $k_{d}=0$, then (115) and (116) are two identities. Moreover (117) degenerates into shape equation (3) of closed lipid bilayers in this case. Otherwise, for small strain, (117) is very close to (3), which may suggest that cross-linking structures have small effects on the shape of lipid bilayers. 
Geometric theory on the elasticity of bio-membranes

It is not hard to verify that $\varepsilon_{12}=0, \varepsilon_{11}=\varepsilon_{22}=\varepsilon$ satisfy (115) and (116) if $\varepsilon$ being a constant. In this case, the sphere with radius $R$ is the solution of (117) if it satisfies

$$
p R^{2}+\left(2 \mu+3 k_{d} \varepsilon\right) R+k_{c} c_{0}\left(c_{0} R-2\right)=0 .
$$

\subsection{Mechanical stabilities of spherical cell membranes}

To discuss the stabilities of spherical cell membranes, we must discuss the second order variations of the functional $\mathcal{F}$. In mathematical point of view presented in section 2 , we must calculate $\delta_{i} \delta_{j} \mathcal{F}(i, j=1,2,3)$. But in physical and symmetric point of view, we just need to calculate $\delta_{3}^{2} \mathcal{F}$ because we can expect that the perturbations along the normal are primary to the instabilities of spherical membranes under the osmotic pressure which is perpendicular to the sphere surfaces.

If we taking $\mathcal{E}_{H}=\frac{k_{c}}{2}\left(2 H+c_{0}\right)^{2}+\mu$ and $\mathcal{E}_{d}=\frac{k_{d}}{2}\left[(2 J)^{2}-Q\right]$, the leading term of (111) is

$$
\delta_{3} \mathcal{F}_{d}=-\frac{k_{d}}{2} \int_{M}\left[\left(a \varepsilon_{11}+2 b \varepsilon_{12}+c \varepsilon_{22}\right)+2 H(2 J)\right] \Omega_{3} d A .
$$

Using Lemmas 3.1, 3.2, Eqs. (98) (100), we can obtain

$$
\begin{aligned}
\delta_{3}^{2} \mathcal{F}_{d}= & -\frac{k_{d}}{2} \int_{M}\left[\left(\delta_{3} a \varepsilon_{11}+2 \delta_{3} b \varepsilon_{12}+\delta_{3} c \varepsilon_{22}\right)+\delta_{3}(2 H)(2 J)\right] \Omega_{3} d A \\
& -\frac{k_{d}}{2} \int_{M}\left[\left(a \delta_{3} \varepsilon_{11}+2 b \delta_{3} \varepsilon_{12}+c \delta_{3} \varepsilon_{22}\right)+2 H \delta_{3}(2 J)\right] \Omega_{3} d A \\
& -\frac{k_{d}}{2} \int_{M}\left[\left(a \varepsilon_{11}+2 b \varepsilon_{12}+c \varepsilon_{22}\right)+2 H(2 J)\right] \Omega_{3} \delta_{3} d A \\
= & k_{d} \int_{M} \frac{3(1+\varepsilon)}{R^{2}} \Omega_{3}^{2} d A-\frac{3 k_{d} \varepsilon}{2} \int_{M} \Omega_{3} \nabla^{2} \Omega_{3} d A,
\end{aligned}
$$

for the spherical cell membrane with radius $R$ and strain $\varepsilon$.

Otherwise, (61) suggests that

$$
\begin{aligned}
\delta_{3}^{2} \mathcal{F}_{c p}= & \int_{M} \Omega_{3}^{2}\left\{k_{c} c_{0}^{2} / R^{2}+2 \mu / R^{2}+2 p / R\right\} d A \\
& +\int_{M} \Omega_{3} \nabla^{2} \Omega_{3}\left\{2 k_{c} c_{0} / R+2 k_{c} / R^{2}-\mu-k_{c} c_{0}^{2} / 2\right\} d A \\
& +\int_{M} k_{c}\left(\nabla^{2} \Omega_{3}\right)^{2} d A .
\end{aligned}
$$

Therefore

$$
\begin{aligned}
\delta_{3}^{2} \mathcal{F}= & \delta_{3}^{2} \mathcal{F}_{d}+\delta_{3}^{2} \mathcal{F}_{c p} \\
= & \int_{M} \Omega_{3}^{2}\left\{3 k_{d} / R^{2}+\left(3 k_{d} \varepsilon+k_{c} c_{0}^{2}+2 \mu\right) / R^{2}+2 p / R\right\} d A \\
& +\int_{M} \Omega_{3} \nabla^{2} \Omega_{3}\left\{2 k_{c} c_{0} / R+2 k_{c} / R^{2}-\left(3 k_{d} \varepsilon+k_{c} c_{0}^{2}+2 \mu\right) / 2\right\} d A \\
& +\int_{M} k_{c}\left(\nabla^{2} \Omega_{3}\right)^{2} d A .
\end{aligned}
$$


If considering (118) and expanding $\Omega_{3}$ as (66), we have

$$
\begin{aligned}
\delta_{3}^{2} \mathcal{F}= & \int_{M} \Omega_{3}^{2}\left\{3 k_{d} / R^{2}+\left(2 k_{c} c_{0} / R^{3}\right)+p / R\right\} d A \\
& +\int_{M} \Omega_{3} \nabla^{2} \Omega_{3}\left\{k_{c} c_{0} / R+2 k_{c} / R^{2}+p R / 2\right\} d A+\int_{M} k_{c}\left(\nabla^{2} \Omega_{3}\right)^{2} d A \\
= & \sum_{l, m}\left|a_{l m}\right|^{2}\left\{3 k_{d}+[l(l+1)-2]\left[l(l+1) k_{c} / R^{2}-k_{c} c_{0} / R-p R / 2\right]\right\} .
\end{aligned}
$$

The zero point of the coefficient of $\left|a_{l m}\right|^{2}$ in above expression is

$$
p_{l}=\frac{6 k_{d}}{[l(l+1)-2] R}+\frac{2 k_{c}\left[l(l+1)-c_{0} R\right]}{R^{3}} \quad(l=2,3, \cdots) .
$$

Obviously, on the one hand, if $k_{d}=0$, (123) is degenerated into (68) with $l \geq 2$. On the other hand, if $k_{d}>0$, we must take the minimum of (123) to obtain the critical pressure.

If let $\xi=l(l+1) \geq 6$, we have

$$
\begin{aligned}
& p(\xi)=\frac{6 k_{d}}{(\xi-2) R}+\frac{2 k_{c}\left(\xi-c_{0} R\right)}{R^{3}}, \\
& \frac{d p}{d \xi}=-\frac{6 k_{d} / R}{(\xi-2)^{2}}+\frac{2 k_{c}}{R^{3}}, \\
& \frac{d^{2} p}{d \xi^{2}}=\frac{12 k_{d} / R}{(\xi-2)^{3}}>0 .
\end{aligned}
$$

$d p / d \xi=0$ and $\xi \geq 6$ imply $\xi=2+R \sqrt{3 k_{d} / k_{c}}$ which is valid only if $3 k_{d} R^{2}>16 k_{c}$. Therefore, the critical pressure is:

$$
p_{c}=\min \left\{p_{l}\right\}=\left\{\begin{array}{c}
\frac{3 k_{d}}{2 R}+\frac{2 k_{c}\left(6-c_{0} R\right)}{R^{3}}<\frac{2 k_{c}\left(10-c_{0} R\right)}{R^{3}} \quad\left(3 k_{d} R^{2}<16 k_{c}\right), \\
\frac{4 \sqrt{3 k_{d} k_{c}}}{R^{2}}+\frac{2 k_{c}}{R^{3}}\left(2-c_{0} R\right) \quad\left(3 k_{d} R^{2}>16 k_{c}\right) .
\end{array}\right.
$$

Eq.(127) includes the classical result for stability of elastic shell. The critical pressure for classical spherical shell is $p_{c} \propto Y h^{2} / R^{2}[32,33]$, where $Y$ is the Young's modulus of the shell. If taking $c_{0}=0, k_{d} \propto Y h, k_{c} \propto Y h^{3}$ and $R \gg h$, our result (127) also gives $p_{c} \propto Y h^{2} / R^{2}$. As far as we know, this is the first time to obtain the critical pressure for spherical shell through the second order variation of free energy without any assumption to the shape of its losing the stability (cf. Ref. [32,33]).

Otherwise, if we take the typical parameters of cell membranes as $k_{c} \sim 20 k_{B} T[4,5]$, $k_{d} \sim 2.4 \mu N / m$ [34], $h \sim 4 n m, R \sim 1 \mu m, c_{0} R \sim 1$, we obtain $p_{c} \sim 4$ Pa from (127), which is much larger than $p_{c} \sim 0.2 \mathrm{~Pa}$ without considering $k_{d}$ induced by the crosslinking structures. Therefore, cross-linking structures greatly enhance the mechanical stabilities of cell membranes.

\section{Conclusion}

In above discussion, we deal with variational problems on closed and open surfaces by using exterior differential forms. We obtain the shape equation of closed lipid bilayers, 
the shape equation and boundary conditions of open lipid bilayers and two-component lipid bilayers, and the shape equation and in-plane stain equations of cell membranes with cross-linking protein structures. Furthermore, we discuss the mechanical stabilities of spherical lipid bilayers and cell membranes.

Some new results are obtained as follows:

(i) The fundamental variational equations in a surface: Eqs. (27) $\sim(36)$.

(ii) The general expressions of the second order variation of the free energy for closed lipid bilayers: theorem 3.3 and Eq. (61).

(iii) The general shape equation and boundary conditions of open lipid bilayers and two-component lipid bilayers: Eqs. (177) $\sim(80)$ and (88) $\sim(91)$.

(iv) The free energy (94), shape equation and strain equations (115) (117) of the cell membranes with cross-linking protein structures.

(v) The critical pressure (127) of losing stabilities for spherical cell membranes. It includes the critical pressures not only for closed lipid bilayers, but also for the classic solid shells. Otherwise it suggests that cross-linking protein structures can enhance the stabilities of cell membranes.

In the future, we will devote ourselves to applying above results to explain the shapes of open lipid bilayers found by Saitoh et al., and predict new shapes of multicomponent lipid bilayers and cell membranes. Moreover, We will discuss whether and how the in-plane modes affect the instability of cell membranes although we believe they have no qualitative effect on our results in section 5.4.

\section{Acknowledgments}

We are grateful to Prof. H W Peng for his useful discussions and to Prof. S S Chern for his advice that we should notice the work by Griffiths et al. Thank Prof. J Guven and G Landolfi for their friendly email discussions. Thank Prof. J Hu, Dr. W Zhao and R An for their kind helps.

\section{Appendix A. Exterior differential forms and Stokes' theorem}

A manifold can be roughly regarded as a multi-dimensional surface. In the neighborhood of every point, we can construct the local coordinates $\left(u^{1}, u^{2}, \cdots, u^{m}\right)$, where $m$ is the dimension of the surface. In this paper we just consider smooth, orientable manifolds and smooth functions.

We call the function $f\left(u^{1}, u^{2}, \cdots, u^{m}\right)$ 0-form and $a_{i}\left(u^{1}, u^{2}, \cdots, u^{m}\right) d u^{i}$ 1-form, where Einstein summation rule is used and it is also used in the following contents. The $r$-form $(r \leq m)$ is defined as $a_{i_{1} i_{2} \cdots i_{r}} d u^{i_{1}} \wedge d u^{i_{2}} \wedge \cdots \wedge d u^{i_{r}}$, where the exterior production " $\wedge$ " satisfies $d u^{i} \wedge d u^{j}=-d u^{j} \wedge d u^{i}$. Denote $\Lambda^{r}=\{$ all $r$-forms $\},(r=0,1,2, \cdots, m)$.

Definition A linear operator $d: \Lambda^{r} \rightarrow \Lambda^{r+1}$ is called the exterior differential operator if it satisfies:

(i) For function $f\left(u^{1}, u^{2}, \cdots, u^{m}\right), d f=\frac{\partial f}{\partial u^{i}} d u^{i}$ is an ordinary differential; 
Geometric theory on the elasticity of bio-membranes

(ii) $d d=0$;

(iii) $\forall \omega_{1} \in \Lambda^{r}$ and $\forall \omega_{2} \in \Lambda^{k}, d\left(\omega_{1} \wedge \omega_{2}\right)=d \omega_{1} \wedge \omega_{2}+(-1)^{r} \omega_{1} \wedge d \omega_{2}$.

Stokes theorem If $\omega$ is an $(m-1)$-form with compact support set on $M$, and $\mathcal{D}$ is a domain with boundary $\partial \mathcal{D}$ in $M$, then

$$
\int_{\mathcal{D}} d \omega=\int_{\partial \mathcal{D}} \omega
$$

\section{Appendix B. Curves in a surface}

If a curve passes through $P$ in the surface, we construct a Frenet frame $\{\mathbf{T}, \mathbf{N}, \mathbf{B}\}$ such that $\mathbf{T}, \mathbf{N}$ and $\mathbf{B}$ are the tangent, normal and binormal vectors of the curve, respectively. Denote $\theta$ the angle between $\mathbf{e}_{1}$ and $\mathbf{T}$. Set $\mathbf{M}=\mathbf{e}_{3} \times \mathbf{T}$. Thus we have

$$
\left\{\begin{array}{l}
\mathbf{T}=\mathbf{e}_{1} \cos \theta+\mathbf{e}_{2} \sin \theta \\
\mathbf{M}=-\mathbf{e}_{1} \sin \theta+\mathbf{e}_{2} \cos \theta
\end{array}\right.
$$

It is not hard to calculate

$$
d \mathbf{T}=\left(d \theta+\omega_{12}\right) \mathbf{M}+\mathbf{e}_{3}\left(\omega_{13} \cos \theta+\omega_{23} \sin \theta\right) .
$$

Frenet Formulas tell us $d \mathbf{T} / d s=\kappa \mathbf{N}$. Therefore, we have the geodesic curvature, the geodesic torsion, and the normal curvature of the curve:

$$
\begin{aligned}
& k_{g}=\kappa \mathbf{N} \cdot \mathbf{M}=(d \mathbf{T} / d s) \cdot \mathbf{M}=\left(d \theta+\omega_{12}\right) / d s, \\
& \tau_{g}=-\left(d \mathbf{e}_{3} / d s\right) \cdot \mathbf{M}=\left[\left(b \omega_{1}+c \omega_{2}\right) \cos \theta-\left(a \omega_{1}+b \omega_{2}\right)(\sin \theta)\right] / d s, \\
& k_{n}=I I / I=\left(a \omega_{1}^{2}+2 b \omega_{1} \omega_{2}+c \omega_{2}^{2}\right) /\left(\omega_{1}^{2}+\omega_{2}^{2}\right) .
\end{aligned}
$$

If the curve along $\mathbf{e}_{1}$ such that $\theta=0$, we have $d s=\omega_{1}, \omega_{2}=0$ and

$$
k_{g}=\omega_{12} / \omega_{1}, \quad \tau_{g}=b, \quad \text { and } \quad k_{n}=a .
$$

\section{Appendix C. Gauss-Bonnet formula}

Using (91), (10) and (13), we have

$$
d \omega_{12}=-K \omega_{1} \wedge \omega_{2} .
$$

This formula was called Theorem Egregium by Gauss. From Theorem Egregium and (B.1), we can derive Gauss-Bonnet formula:

$$
\int_{M} K d A+\int_{C} k_{g} d s=2 \pi \chi(M)
$$

where $\chi(M)$ is the characteristic number of smooth surface $M$ with smooth edge $C$. $\chi(M)=1$ for a simple surface with an edge. For a closed surface, we have

$$
\int_{M} K d A=2 \pi \chi(M)
$$


Geometric theory on the elasticity of bio-membranes

\section{Appendix D. The tensor expressions of $\nabla, \bar{\nabla}, \tilde{\nabla}, \nabla^{2}, \nabla \cdot \bar{\nabla}$, and $\nabla \cdot \tilde{\nabla}$}

At every point $\mathbf{r}$ in the surface, we can take local coordinates $\left(u^{1}, u^{2}\right)$ where the first and the second fundamental form are denoted by $I=g_{i j} d u^{i} d u^{j}$ and $I I=L_{i j} d u^{i} d u^{j}$, respectively. Let $\left(g^{i j}\right)=\left(g_{i j}\right)^{-1},\left(L^{i j}\right)=\left(L_{i j}\right)^{-1}$ and $\mathbf{r}_{i}=\partial \mathbf{r} / \partial u^{i}$, thus we have

$$
\begin{aligned}
& \nabla=g^{i j} \mathbf{r}_{i} \frac{\partial}{\partial u^{j}}, \\
& \bar{\nabla}=\mathbf{r}_{i}\left(2 H g^{i j}-K L^{i j}\right) \frac{\partial}{\partial u^{j}}, \\
& \tilde{\nabla}=K L^{i j} \mathbf{r}_{i} \frac{\partial}{\partial u^{j}}, \\
& \nabla^{2}=\frac{1}{\sqrt{g}} \frac{\partial}{\partial u^{i}}\left(\sqrt{g} g^{i j} \frac{\partial}{\partial u^{j}}\right), \\
& \nabla \cdot \bar{\nabla}=\frac{1}{\sqrt{g}} \frac{\partial}{\partial u^{i}}\left[\sqrt{g}\left(2 H g^{i j}-K L^{i j}\right) \frac{\partial}{\partial u^{j}}\right], \\
& \nabla \cdot \tilde{\nabla}=\frac{1}{\sqrt{g}} \frac{\partial}{\partial u^{i}}\left(\sqrt{g} K L^{i j} \frac{\partial}{\partial u^{j}}\right) .
\end{aligned}
$$

As an example, We will prove the last one of above expressions.

Proof: If taking the orthogonal local coordinates, we have $I=g_{11}\left(d u^{1}\right)^{2}+g_{22}\left(d u^{2}\right)^{2}=$ $\omega_{1}^{2}+\omega_{2}^{2}$, which implies $\omega_{1}=\sqrt{g_{11}} d u^{1}$ and $\omega_{2}=\sqrt{g_{22}} d u^{2}$. For function $f$, on the one hand, we have $d f\left(u^{1}, u^{2}\right)=f_{1} \omega_{1}+f_{2} \omega_{2}=f_{1} \sqrt{g_{11}} d u^{1}+f_{2} \sqrt{g_{22}} d u^{2}$, on the other hand, we have $d f=\frac{\partial f}{\partial u^{1}} d u^{1}+\frac{\partial f}{\partial u^{2}} d u^{2}$. Therefore, $f_{1}=\frac{1}{\sqrt{g_{11}}} \frac{\partial f}{\partial u^{1}}, \quad f_{2}=\frac{1}{\sqrt{g_{22}}} \frac{\partial f}{\partial u^{2}}$.

The second fundamental form $I I=a \omega_{1}^{2}+2 b \omega_{1} \omega_{2}+c \omega_{1}^{2}=L_{i j} d u^{i} d u^{j}$ implies $a=L_{11} / g_{11}, \quad b=L_{12} / \sqrt{g}, \quad c=L_{22} / g_{22}$. Thus $K=a c-b^{2}=\left(L_{11} L_{22}-L_{12}^{2}\right) / g$, and

$$
\begin{aligned}
& L^{11}=\frac{L_{22}}{L_{11} L_{22}-L_{12}^{2}} \Rightarrow L_{22}=g K L^{11} ; \\
& L^{12}=-\frac{L_{12}}{L_{11} L_{22}-L_{12}^{2}} \Rightarrow L_{12}=-g K L^{12} ; \\
& L^{22}=\frac{L_{11}}{L_{11} L_{22}-L_{12}^{2}} \Rightarrow L_{11}=g K L^{22} .
\end{aligned}
$$

Moreover, we have

$$
\begin{aligned}
\tilde{*} \tilde{d} f= & -f_{2} \omega_{13}+f_{1} \omega_{23}=-f_{2}\left(a \omega_{1}+b \omega_{2}\right)+f_{2}\left(b \omega_{1}+c \omega_{2}\right) \\
= & \frac{1}{\sqrt{g}}\left(L_{12} \frac{\partial f}{\partial u^{1}}-L_{11} \frac{\partial f}{\partial u^{2}}\right) d u^{1}+\frac{1}{\sqrt{g}}\left(L_{22} \frac{\partial f}{\partial u^{1}}-L_{12} \frac{\partial f}{\partial u^{2}}\right) d u^{2} \\
d \tilde{*} \tilde{d} f= & \left\{\frac{\partial}{\partial u^{1}}\left[\sqrt{g} K\left(L^{11} \frac{\partial f}{\partial u^{1}}+L^{12} \frac{\partial f}{\partial u^{2}}\right)\right]\right. \\
& \left.+\frac{\partial}{\partial u^{2}}\left[\sqrt{g} K\left(L^{12} \frac{\partial f}{\partial u^{1}}+L^{22} \frac{\partial f}{\partial u^{2}}\right)\right]\right\} d u^{1} \wedge d u^{2} .
\end{aligned}
$$

Therefore, $\nabla \cdot \tilde{\nabla} f=\frac{d \tilde{*} \tilde{d} f}{\omega_{1} \wedge \omega_{2}}=\frac{1}{\sqrt{g}} \frac{\partial}{\partial u^{i}}\left(\sqrt{g} K L^{i j} \frac{\partial f}{\partial u^{j}}\right)$. 
Geometric theory on the elasticity of bio-membranes

\section{References}

[1] Edidin M 2003 Nature Reviews Molecular Cell Biology 4414

[2] Singer S J and Nicolson G L 1972 Science $\mathbf{1 7 5} 720$

[3] Helfrich W 1973 Z. Naturforsch. C 28693

[4] Duwe H P, Kaes J and Sackmann E 1990 J. Phys. Fr. 51945

[5] Mutz M and Helfrich W 1990 J. Phys. Fr. 51991

[6] Ou-Yang Z C, Liu J X and Xie Y Z 1999 Geometric Methods in the Elastic Theory of Membranes in Liquid Cristal Phases (Singapore: World Scientific)

[7] Lipowsky R 1991 Nature 349 475; Seifert U 1997 Adv. Phys. 4613

[8] Ou-Yang Z C and Helfrich W 1987 Phys. Rev. Lett. 59 2486; 1989 Phys. Rev. A 395280

[9] Saitoh A, Takiguchi K, Tanaka Y and Hotani H 1998 Proc. Natl. Acad. Sci. 951026

[10] Nomura F, Nagata M, Inaba T, Hiramatsu H, Hotani H and Takiguchi K 2001 Proc. Natl. Acad. Sci. 982340

[11] Capovilla R, Guven J and Santiago J A 2002 Phys. Rev. E 66021607

[12] Capovilla R and Guven J 2002 J. Phys. A: Math. Gen. 356233

[13] Tu Z C and Ou-Yang Z C 2003 Phys. Rev. E 68 061915; An R and Tu Z C 2003 Prerint math-phys/0307007

[14] Chiras D 2002 Human Biology: Health, Homeostasis, and the Environment, 4th ed. (Boston: Jones \& Bartlett Publishers)

[15] Griffiths P 1983 Exterior Differential Systems and the Calculus of Variations (Boston: Birkhäuser)

[16] Bryant R, Chern S S, Gardner R, Goldschmidt H and Griffiths P 1991 Exterior Differential Systems (New York: Springer-Verlag)

[17] Kamien R D 2002 Rev. Mod. Phys. 74953

[18] Chern S S and Chen W H 2001 Lectures on Differential Geometry 2nd. (Beijing: Peking University Press)

[19] Westenholz C V 1981 Differential Forms in Mathematical Physics (Amsterdam: North-Holland)

[20] Natio H, Okuda M and Ou-Yang Z C 1995 Phys. Rev. E 522095

[21] Capovilla R and Guven J 2004 J. Phys. A: Math. Gen. 375983

[22] Landolfi G 2003 J. Phys. A: Math. Gen. 3611937

[23] Alexandrov A D 1962 Amer. Math. Soc. Transl. 21341

[24] Naito H, Okuda M and Ou-Yang Z C 1993 Phys. Rev. E 482304

[25] Ou-Yang Z C 1990 Phys. Rev. A 414517

[26] Wang Z X and Guo D R 2000 Introduction to Special Function (Beijing: Peking University Press)

[27] Jülicher F and Lipowsky R 1996 Phys. Rev. E 532670

[28] Baumgart T, Hess S T and Webb W W 2003 Nature 425821

[29] Treloar L R G 1975 The Physics of Rubber Elasticity (Oxford: Clarendon Press)

[30] Dio M and Edwards S F 1986 The Theory of protein chain Dynamics (Oxford: Clarendon Press)

[31] Wu J K and Wang M Z 1981 Introduction to Elastic theory (Beijing: Peking University Press); Wu J K and Su X Y 1994 Stabilities of Elastic Systems (Beijing: Science Press)

[32] Landau L D and Lifshitz E M 1997 Theory of Elasticity 3rd edn. (Oxford: ButterworthHeinemann)

[33] Pogorelov A V 1989 Bendings of surfaces and stability of shells (Providence, R.I.: AMS)

[34] Lenormand G et al. 2001 Biophys. J. 8143 